\title{
Hopf Bifurcation Analysis for a Computer Virus Model with Two Delays
}

\author{
Zizhen Zhang ${ }^{1,2}$ and Huizhong Yang ${ }^{1}$ \\ ${ }^{1}$ Key Laboratory of Advanced Process Control for Light Industry, Jiangnan University, Wuxi 214122, China \\ ${ }^{2}$ School of Management Science and Engineering, Anhui University of Finance and Economics, Bengbu 233030, China \\ Correspondence should be addressed to Huizhong Yang; yhz@jiangnan.edu.cn
}

Received 18 August 2013; Accepted 26 August 2013

Academic Editor: Luca Guerrini

Copyright (c) 2013 Z. Zhang and H. Yang. This is an open access article distributed under the Creative Commons Attribution License, which permits unrestricted use, distribution, and reproduction in any medium, provided the original work is properly cited.

This paper is concerned with a computer virus model with two delays. Its dynamics are studied in terms of local stability and Hopf bifurcation. Sufficient conditions for local stability of the positive equilibrium and existence of the local Hopf bifurcation are obtained by regarding the possible combinations of the two delays as a bifurcation parameter. Furthermore, explicit formulae for determining direction of the Hopf bifurcation and stability of the bifurcating periodic solutions are obtained by using the normal form method and center manifold theory. Finally, some numerical simulations are presented to support the theoretical results.

\section{Introduction}

Since the pioneering work of Kephart and White $[1,2]$, many classical epidemic models such as SIR [3-5], SIRS [68], SEIR $[9,10]$, and SEIRS [11, 12], SEIQV [13] have been used to describe the spread of a computer virus in computer network due to the high similarity between computer viruses and biological viruses. In [9], Yuan and Chen proposed the following SEIR model:

$$
\begin{gathered}
\frac{d S(t)}{d t}=\mu N-v(t) S(t)-\rho_{S R} S(t)-\mu S(t), \\
\frac{d E(t)}{d t}=v(t) S(t)-m_{E} \alpha E(t), \\
\frac{d I(t)}{d t}=\alpha E(t)-(\gamma+\mu) I(t), \\
\frac{d R(t)}{d t}=\rho_{S R} S(t)+\rho_{E R} E(t)+\gamma I(t)-\mu R(t),
\end{gathered}
$$

where $S(t), E(t), I(t)$, and $R(t)$ denote the numbers of nodes at time $t$ in states susceptible, exposed, infectious, and recovered, respectively. Yuan and Chen [9] studied the behaviors of virus propagation with the presence of antivirus countermeasures by analyzing the equilibrium stability of system (1).

It is well known that time delays of one or other reasons can cause a stable equilibrium to become unstable and make a system bifurcate periodic solutions and dynamical systems with delay have been studied by many scholars [14-23] Starting from this point and considering that the antivirus software may use a period to clean the viruses in a computer, Dong et al. [10] proposed the following model with delay:

$$
\begin{gathered}
\frac{d S(t)}{d t}=\mu N-\beta I(t) S(t)-\left(\rho_{S R}+\mu\right) S(t), \\
\frac{d E(t)}{d t}=\beta I(t) S(t)-(\alpha+\mu) E(t)-\rho_{E R} E(t-\tau), \\
\frac{d I(t)}{d t}=\alpha E(t)-(\gamma+\mu) I(t), \\
\frac{d R(t)}{d t}=\rho_{S R} S(t)+\rho_{E R} E(t-\tau)+\gamma I(t-\tau)-\mu R(t),
\end{gathered}
$$

where $N$ is the total number of computers in a network. $\mu$ describes the impact of quarantine or replacement. $\rho_{S R}$ describes the impact of implementing real-time immunization. $\rho_{E R}$ describes the impact of cleaning the virus and 
immunizing the computers. $\beta$ is the transmission coefficient. $\alpha$ and $\gamma$ are the state transition rates. And $\tau$ is the period which a computer uses antivirus software to clean viruses. Dong et al. [10] discussed the local stability and existence of local Hopf bifurcation of system (2). Properties of the Hopf bifurcation were also investigated in [10].

However, Yuan and Chen [9] Dong et al. [10] supposed that the recovered computers have a permanent immunization period and can no longer be infected. This is not consistent with real situation. In order to overcome limitation and considering that the recovered computers may be infected again after a temporary immunity period, we investigate the following system with two delays in this paper:

$$
\begin{gathered}
\frac{d S(t)}{d t}=\mu N-\beta I(t) S(t)-\left(\rho_{S R}+\mu\right) S(t)+\varepsilon R\left(t-\tau_{2}\right), \\
\frac{d E(t)}{d t}=\beta I(t) S(t)-(\alpha+\mu) E(t)-\rho_{E R} E\left(t-\tau_{1}\right), \\
\frac{d I(t)}{d t}=\alpha E(t)-(\gamma+\mu) I(t), \\
\frac{d R(t)}{d t}=\rho_{S R} S(t)+\rho_{E R} E\left(t-\tau_{1}\right)+\gamma I\left(t-\tau_{1}\right) \\
-\mu R(t)-\varepsilon R\left(t-\tau_{2}\right),
\end{gathered}
$$

where $\tau_{1}$ is the period that a computer uses antivirus software to clean viruses and $\tau_{2}$ is the temporary immunity period after which a recovered computer may be infected again. $\varepsilon$ is the transition rate from $R$ to $S$.

This paper is organized as follows. In Section 2, local stability of the positive equilibrium and the existence of local Hopf bifurcation are discussed. In Section 3, the direction of the Hopf bifurcation and the stability of the bifurcating periodic solutions are determined by using the normal form theory and center manifold theorem. In order to illustrate the validity of the theoretical analysis, some numerical simulations are presented in Section 4. Some main conclusions are drawn in Section 5.

\section{Stability and Existence of Local Hopf Bifurcation}

In this section, we will study the stability of positive equilibrium and the existence of Hopf bifurcation. It is not difficult to verify if

$$
R_{0}=\frac{\alpha \beta \mu N(\mu+\varepsilon)+\varepsilon \rho_{S R}(\mu+\gamma)\left(\alpha+\mu+\rho_{E R}\right)}{(\mu+\varepsilon)(\mu+\gamma)\left(\mu+\rho_{S R}\right)\left(\alpha+\mu+\rho_{E R}\right)}>1
$$

system (3) has a unique positive equilibrium $D_{*}\left(S_{*}, E_{*}, I_{*}\right.$, $R_{*}$ ), where

$$
\begin{gathered}
S_{*}=\frac{(\mu+\gamma)\left(\alpha+\mu+\rho_{E R}\right)}{\alpha \beta}, \\
I_{*}=\left(\alpha \beta \mu N(\mu+\varepsilon)+\varepsilon \rho_{S R}(\mu+\gamma)\left(\alpha+\mu+\rho_{E R}\right)\right. \\
\left.-(\mu+\varepsilon)(\mu+\gamma)\left(\mu+\rho_{S R}\right)\left(\alpha+\mu+\rho_{E R}\right)\right) \\
\times\left(\alpha \beta \mu(\mu+\varepsilon+\gamma)+\beta \mu(\mu+\gamma)\left(\mu+\varepsilon+\rho_{E R}\right)\right)^{-1},
\end{gathered}
$$

$$
\begin{gathered}
E_{*}=\frac{\mu+\gamma}{\alpha} I_{*}, \\
R_{*}=\frac{\rho_{S R}(\mu+\gamma)\left(\alpha+\mu+\rho_{E R}\right)+\left[\alpha \beta \gamma+\beta \rho_{E R}(\mu+\gamma)\right] I_{*}}{\alpha \beta(\mu+\varepsilon)} .
\end{gathered}
$$

$R_{0}$ is called the basic reproduction number.

Linearizing system (3) at the positive equilibrium $D_{*}$ yields the following linear system:

$$
\begin{aligned}
& \frac{d S(t)}{d t}=a_{11} S(t)+a_{13} I(t)+c_{14} R\left(t-\tau_{2}\right), \\
& \frac{d E(t)}{d t}=a_{21} S(t)+a_{22} E(t)+a_{23} I(t)+b_{22} E\left(t-\tau_{1}\right), \\
& \frac{d I(t)}{d t}=a_{32} E(t)+a_{33} I(t), \\
& \frac{d R(t)}{d t}=a_{41} S(t)+a_{44} R(t)+b_{42} E\left(t-\tau_{1}\right)+b_{43} I\left(t-\tau_{1}\right) \\
& \quad+c_{44} R\left(t-\tau_{2}\right),
\end{aligned}
$$

where

$$
\begin{gathered}
a_{11}=-\left(\mu+\rho_{S R}+\beta I_{*}\right), \quad a_{13}=-\beta S_{*}, \\
a_{21}=\beta I_{*}, \quad a_{22}=-(\alpha+\mu), \quad a_{23}=\beta S_{*}, \\
a_{32}=\alpha, \quad a_{33}=-(\mu+\gamma), \\
a_{41}=\rho_{S R}, \quad a_{44}=-\mu, \\
b_{22}=-\rho_{E R}, \quad b_{42}=\rho_{E R}, \quad b_{43}=\gamma, \\
c_{14}=\varepsilon, \quad c_{44}=-\varepsilon .
\end{gathered}
$$

Thus, the characteristic equation of system (3) at $D_{*}$ is

$$
\begin{aligned}
\lambda^{4}+ & A_{3} \lambda^{3}+A_{2} \lambda^{2}+A_{1} \lambda+A_{0} \\
& +\left(B_{3} \lambda^{3}+B_{2} \lambda^{2}+B_{1} \lambda+B_{0}\right) e^{-\lambda \tau_{1}} \\
& +\left(C_{3} \lambda^{3}+C_{2} \lambda^{2}+C_{1} \lambda+C_{0}\right) e^{-\lambda \tau_{2}} \\
& +\left(D_{2} \lambda^{2}+D_{1} \lambda+D_{0}\right) e^{-\lambda\left(\tau_{1}+\tau_{2}\right)}=0
\end{aligned}
$$

where

$$
\begin{gathered}
A_{0}=a_{11} a_{44}\left(a_{22} a_{33}-a_{23} a_{32}\right)+a_{13} a_{21} a_{32} a_{44}, \\
A_{1}=a_{23} a_{32}\left(a_{11}+a_{44}\right)-a_{11} a_{22}\left(a_{33}+a_{44}\right)-a_{13} a_{21} a_{32} \\
-a_{33} a_{44}\left(a_{11}+a_{22}\right), \\
A_{2}=\left(a_{11}+a_{22}\right)\left(a_{33}+a_{44}\right)+a_{11} a_{22}+a_{33} a_{44}-a_{23} a_{32}, \\
A_{3}=-\left(a_{11}+a_{22}+a_{33}+a_{44}\right), \quad B_{0}=a_{11} a_{33} a_{44} b_{22}, \\
B_{1}=-a_{11} a_{33} b_{22}-a_{44} b_{22}\left(a_{11}+a_{33}\right),
\end{gathered}
$$




$$
\begin{gathered}
B_{2}=b_{22}\left(a_{11}+a_{33}+a_{44}\right), \quad B_{3}=-b_{22}, \\
C_{0}=\left(a_{22} a_{33}-a_{23} a_{32}\right)\left(a_{11} c_{44}-a_{41} c_{14}\right)+a_{13} a_{21} a_{32} c_{44}, \\
C_{1}=a_{41} c_{14}\left(a_{22}+a_{33}\right)+c_{44}\left(a_{23} a_{32}-a_{11} a_{22}\right) \\
-a_{33} a_{44}\left(a_{11}+a_{22}\right), \\
C_{2}=c_{44}\left(a_{11}+a_{22}+a_{33}\right)-a_{41} c_{14}, \\
C_{3}=-c_{44}, \quad D_{2}=b_{22} c_{44}, \\
D_{1}=c_{14}\left(a_{41} b_{22}-a_{21} b_{42}\right)-b_{22} c_{44}\left(a_{11}+a_{33}\right) . \\
D_{0}=a_{33}\left(a_{11} b_{22} c_{44}+a_{21} b_{42} c_{14}\right) \\
\quad-c_{14}\left(a_{21} a_{32} b_{43}+a_{33} a_{41} b_{22}\right) .
\end{gathered}
$$

Case $1\left(\tau_{1}=\tau_{2}=0\right)$. Equation $(8)$ reduces to

$$
\lambda^{4}+A_{13} \lambda^{3}+A_{12} \lambda^{2}+A_{11} \lambda+A_{10}=0,
$$

where

$$
\begin{gathered}
A_{10}=A_{0}+B_{0}+C_{0}+D_{0}, \quad A_{11}=A_{1}+B_{1}+C_{1}+D_{1}, \\
A_{12}=A_{2}+B_{2}+C_{2}+D_{2}, \quad A_{13}=A_{3}+B_{3}+C_{3} .
\end{gathered}
$$

By the Routh-Hurwitz criterion, sufficient conditions for all roots of (10) to have a negative real part are given in the following form:

$$
\begin{gathered}
\operatorname{Det}_{1}=A_{13}>0, \\
\operatorname{Det}_{2}=\left|\begin{array}{cc}
A_{13} & 1 \\
A_{11} & A_{12}
\end{array}\right|>0, \\
\operatorname{Det}_{3}=\left|\begin{array}{ccc}
A_{13} & 1 & 0 \\
A_{11} & A_{12} & A_{13} \\
0 & A_{10} & A_{11}
\end{array}\right|>0, \\
\operatorname{Det}_{4}=\left|\begin{array}{cccc}
A_{13} & 1 & 0 & 0 \\
A_{11} & A_{12} & A_{13} & 1 \\
0 & A_{10} & A_{11} & A_{12} \\
0 & 0 & 0 & A_{10}
\end{array}\right|>0 .
\end{gathered}
$$

Thus, if condition $\left(H_{1}\right)$ (12) holds, then the positive equilibrium $D_{*}$ is locally asymptotically stable in the absence of delay.

Case $2\left(\tau_{1}>0, \tau_{2}=0\right)$. When $\tau_{1}>0, \tau_{2}=0$, (8) becomes the following form:

$$
\begin{aligned}
\lambda^{4}+ & A_{23} \lambda^{3}+A_{22} \lambda^{2}+A_{21} \lambda+A_{20} \\
& +\left(B_{23} \lambda^{3}+B_{22} \lambda^{2}+B_{21} \lambda+B_{20}\right) e^{-\lambda \tau_{1}}=0,
\end{aligned}
$$

where

$$
\begin{array}{cc}
A_{23}=A_{3}+C_{3}, & A_{22}=A_{2}+C_{2}, \\
A_{21}=A_{1}+C_{1}, & A_{20}=A_{0}+C_{0}, \\
B_{23}=B_{3}, & B_{22}=B_{2}+D_{2}, \\
B_{21}=B_{1}+D_{1}, & B_{20}=B_{0}+D_{0} .
\end{array}
$$

Let $\lambda=i \omega_{1}\left(\omega_{1}>0\right)$ be the root of (13). Then, we can get

$$
\begin{aligned}
& \left(B_{21} \omega_{1}-B_{23} \omega_{1}^{3}\right) \sin \tau_{1} \omega_{1}+\left(B_{20}-B_{22} \omega_{1}^{2}\right) \cos \tau_{1} \omega_{1} \\
& =A_{22} \omega_{1}^{2}-\omega_{1}^{4}-A_{20}, \\
& \left(B_{21} \omega_{1}-B_{23} \omega_{1}^{3}\right) \cos \tau_{1} \omega_{1}-\left(B_{20}-B_{22} \omega_{1}^{2}\right) \sin \tau_{1} \omega_{1} \\
& =A_{23} \omega_{1}^{3}-A_{21} \omega_{1},
\end{aligned}
$$

which follows that

$$
\omega_{1}^{8}+e_{23} \omega_{1}^{6}+e_{22} \omega_{1}^{4}+e_{21} \omega_{1}^{2}+e_{20}=0,
$$

where

$$
\begin{gathered}
e_{20}=A_{20}^{2}-B_{20}^{2}, \\
e_{21}=A_{21}^{2}-B_{21}^{2}-2 A_{20} A_{22}+2 B_{20} B_{22}, \\
e_{22}=A_{22}^{2}-B_{22}^{2}-2 A_{21} A_{23}+2 A_{20}+2 B_{21} B_{23}, \\
e_{23}=A_{23}^{2}-B_{23}^{2}-2 A_{22} .
\end{gathered}
$$

Let $\omega_{1}^{2}=v_{1}$; then, (16) becomes

$$
v_{1}^{4}+e_{23} v_{1}^{3}+e_{22} v_{1}^{2}+e_{21} v_{1}+e_{20}=0 .
$$

Discussion about the roots of (18) is similar to that in [15]. Therefore, we have the following lemma.

Lemma 1. Let

$$
\begin{gathered}
f_{1}\left(v_{1}\right)=v_{1}^{4}+e_{23} v_{1}^{3}+e_{22} v_{1}^{2}+e_{21} v_{1}+e_{20} . \\
p=\frac{1}{2} e_{22}-\frac{3}{16} e_{23}^{2}, \quad q=\frac{1}{32} e_{23}^{3}-\frac{1}{8} e_{22} e_{23}+e_{21}, \\
\alpha_{1}=\left(\frac{q}{2}\right)^{3}+\left(\frac{p}{3}\right)^{3}, \quad \beta_{1}=-\frac{1}{2}+\frac{\sqrt{3}}{2} i \\
y_{1}=\sqrt[3]{-\frac{q}{2}+\sqrt{\alpha_{1}}}+\sqrt[3]{-\frac{q}{2}-\sqrt{\alpha_{1}}}, \\
y_{2}=\beta_{1} \sqrt[3]{-\frac{q}{2}+\sqrt{\alpha_{1}}}+\beta_{1}^{2} \sqrt[3]{-\frac{q}{2}-\sqrt{\alpha_{1}}} \\
y_{3}=\beta_{1}^{2} \sqrt[3]{-\frac{q}{2}+\sqrt{\alpha_{1}}}+\beta_{1} \sqrt[3]{-\frac{q}{2}-\sqrt{\alpha_{1}}} \\
v_{1 i}=y_{i}-\frac{3 e_{23}}{4}, \quad i=1,2,3 .
\end{gathered}
$$


Then, for (18), one has the following:

(i) if $e_{20}<0$, (18) has at least one positive root;

(ii) if $e_{20} \geq 0$ and $\alpha_{1} \geq 0$, (18) has positive roots if and only if $v_{11}>0$ and $f_{1}\left(v_{11}\right)<0$;

(iii) if $e_{20} \geq 0$ and $\alpha_{1}<0$, (18) has positive roots if and only if there exists at least one $v_{1 *} \in\left\{v_{11}, v_{12}, v_{13}\right\}$ such that $v_{1 *}>0$ and $f_{1}\left(v_{1 *}\right) \leq 0$.

In what follows, we assume that $\left(H_{21}\right)$ : the coefficients in $f_{1}\left(v_{1}\right)$ satisfy one of the following conditions in $(a)-(c)$ :

(a) $e_{20}<0$;

(b) $e_{20} \geq 0, \alpha_{1} \geq 0, v_{11}>0$, and $f_{1}\left(v_{11}\right)<0$;

(c) $e_{20} \geq 0, \alpha_{1}<0$, and there exists at least one $v_{1 *} \epsilon$ $\left\{v_{11}, v_{12}, v_{13}\right\}$ such that $v_{1 *}>0$ and $f_{1}\left(v_{1 *}\right) \leq 0$.

If $\left(H_{21}\right)$ holds, we know that (16) has at least a positive root $\omega_{10}$ such that (13) has a pair of purely imaginary root $\pm i \omega_{10}$. The corresponding critical value of the delay is

$$
\begin{aligned}
\tau_{1 k}= & \frac{1}{\omega_{10}} \arccos \frac{\mathrm{p}_{6} \omega_{10}^{6}+\mathrm{p}_{4} \omega_{10}^{4}+\mathrm{p}_{2} \omega_{10}^{2}+\mathrm{p}_{0}}{\mathrm{q}_{6} \omega_{10}^{6}+\mathrm{q}_{4} \omega_{10}^{4}+\mathrm{q}_{2} \omega_{10}^{2}+\mathrm{q}_{0}} \\
& +\frac{2 k \pi}{\omega_{10}}, \quad k=0,1,2, \ldots,
\end{aligned}
$$

where

$$
\begin{gathered}
p_{0}=-A_{20} B_{20}, \quad p_{2}=A_{20} B_{22}-A_{21} B_{21}+A_{22} B_{20}, \\
p_{4}=A_{21} B_{23}-A_{22} B_{22}+A_{23} B_{21}-B_{20}, \\
p_{6}=B_{22}-A_{23} B_{23}, \quad q_{0}=B_{20}^{2}, \\
q_{2}=B_{21}^{2}-2 B_{20} B_{22}, \\
q_{4}=B_{22}^{2}-2 B_{21} B_{23}, \quad q_{6}=B_{23}^{2} .
\end{gathered}
$$

Differentiating both sides of (13) regarding $\tau_{1}$, we can obtain

$$
\begin{aligned}
{\left[\frac{d \lambda}{d \tau_{1}}\right]^{-1}=} & -\frac{4 \lambda^{3}+3 A_{23} \lambda^{2}+2 A_{22} \lambda+A_{21}}{\lambda^{5}+A_{23} \lambda^{4}+A_{22} \lambda^{3}+A_{21} \lambda^{2}+A_{20} \lambda} \\
& +\frac{3 B_{23} \lambda^{2}+2 B_{22} \lambda+B_{21}}{B_{23} \lambda^{4}+B_{22} \lambda^{3}+B_{21} \lambda^{2}+B_{20} \lambda}-\frac{\tau_{1}}{\lambda} .
\end{aligned}
$$

Thus,

$$
\begin{aligned}
\operatorname{Re}\left[\frac{d \lambda}{d \tau}\right]_{\tau_{1}=\tau_{10}}^{-1} & \\
= & \left(4 \omega_{10}^{6}+3\left(A_{23}^{2}-2 A_{22}\right) \omega_{10}^{4}\right. \\
& \left.+2\left(A_{22}^{2}+2 A_{20}-2 A_{21} A_{23}\right) \omega_{10}^{2}+A_{21}^{2}-2 A_{20} A_{22}\right)
\end{aligned}
$$

$$
\begin{aligned}
& \times\left(\omega_{10}^{8}+\left(A_{23}^{2}-2 A_{22}\right) \omega_{10}^{6}\right. \\
& +\left(A_{22}^{2}+2 A_{20}-2 A_{21} A_{23}\right) \omega_{10}^{4} \\
& \left.+\left(A_{21}^{2}-2 A_{20} A_{22}\right) \omega_{10}^{2}+A_{20}^{2}\right)^{-1} \\
& -\left(3 B_{23}^{2} \omega_{10}^{4}+2\left(B_{22}^{2}-2 B_{21} B_{23}\right) \omega_{10}^{2}\right. \\
& \left.\quad+B_{21}^{2}-2 B_{20} B_{22}\right) \\
& \times\left(B_{23}^{2} \omega_{10}^{6}+\left(B_{22}^{2}-2 B_{21} B_{23}\right) \omega_{10}^{4}\right. \\
& \left.\quad+\left(B_{21}^{2}-2 B_{20} B_{22}\right) \omega_{10}^{2}+B_{20}^{2}\right)^{-1} .
\end{aligned}
$$

From (16), we can get

$$
\begin{aligned}
& \operatorname{Re}\left[\frac{d \lambda}{d \tau}\right]_{\tau_{1}=\tau_{10}}^{-1} \\
&=f_{1}^{\prime}\left(v_{1}^{*}\right) \times\left(B_{23}^{2} \omega_{10}^{6}+\left(B_{22}^{2}-2 B_{21} B_{23}\right) \omega_{10}^{4}\right. \\
&\left.+\left(B_{21}^{2}-2 B_{20} B_{22}\right) \omega_{10}^{2}+B_{20}^{2}\right)^{-1}
\end{aligned}
$$

where $v_{1}^{*}=\omega_{10}^{2}$.

Thus, if condition $\left(H_{22}\right) f_{1}^{\prime}\left(v_{1}^{*}\right) \neq 0$ holds, then the transversality condition is satisfied. According to the Hopf bifurcation theorem in [24], we have the following results.

Theorem 2. Suppose that the conditions $\left(H_{21}\right)-\left(H_{22}\right)$ hold. Consider

(i) the positive equilibrium $D_{*}\left(S_{*}, E_{*}, I_{*}, R_{*}\right)$ of system (3) is asymptotically stable for $\tau_{1} \in\left[0, \tau_{10}\right)$;

(ii) system (3) undergoes a Hopf bifurcation at the positive equilibrium $D_{*}\left(S_{*}, E_{*}, I_{*}, R_{*}\right)$ when $\tau_{1}=\tau_{10}$ and $a$ family of periodic solutions bifurcate from the positive equilibrium $D_{*}\left(S_{*}, E_{*}, I_{*}, R_{*}\right)$ near $\tau_{1}=\tau_{10}$.

Case $3\left(\tau_{1}=0, \tau_{2}>0\right)$. When $\tau_{1}=0, \tau_{2}>0$, (8) becomes the following form:

$$
\begin{aligned}
\lambda^{4}+ & A_{33} \lambda^{3}+A_{32} \lambda^{2}+A_{31} \lambda+A_{30} \\
& +\left(C_{33} \lambda^{3}+C_{32} \lambda^{2}+C_{31} \lambda+C_{30}\right) e^{-\lambda \tau_{2}}=0,
\end{aligned}
$$

where

$$
\begin{array}{cc}
A_{33}=A_{3}+B_{3}, & A_{32}=A_{2}+B_{2}, \\
A_{31}=A_{1}+B_{1}, & A_{30}=A_{0}+B_{0}, \\
C_{33}=C_{33}, & C_{32}=C_{2}+D_{2}, \\
C_{31}=C_{1}+D_{1}, & C_{30}=C_{0}+D_{0} .
\end{array}
$$


Let $\lambda=i \omega_{2}\left(\omega_{2}>0\right)$ be the root of (25), and we have

$$
\begin{aligned}
& \left(C_{31} \omega_{2}-C_{33} \omega_{2}^{3}\right) \sin \tau_{2} \omega_{2}+\left(C_{30}-C_{32} \omega_{2}^{2}\right) \cos \tau_{2} \omega_{2} \\
& \quad=A_{32} \omega_{2}^{2}-\omega_{2}^{4}-A_{30}, \\
& \left(C_{31} \omega_{2}-C_{33} \omega_{2}^{3}\right) \cos \tau_{2} \omega_{2}-\left(C_{30}-C_{32} \omega_{2}^{2}\right) \sin \tau_{2} \omega_{2} \\
& =A_{33} \omega_{2}^{3}-A_{31} \omega_{2},
\end{aligned}
$$

from which, we can obtain

$$
\omega_{2}^{8}+e_{33} \omega_{2}^{6}+e_{32} \omega_{2}^{4}+e_{31} \omega_{2}^{2}+e_{30}=0
$$

where

$$
\begin{gathered}
e_{30}=A_{30}^{2}-C_{30}^{2}, \\
e_{31}=A_{31}^{2}-C_{31}^{2}-2 A_{30} A_{32}+2 C_{20} C_{32}, \\
e_{32}=A_{32}^{2}-B_{32}^{2}-2 A_{31} A_{33}+2 A_{30}+2 C_{31} C_{33}, \\
e_{33}=A_{33}^{2}-C_{33}^{2}-2 A_{32} .
\end{gathered}
$$

Let $\omega_{2}^{2}=v_{2}$, then (28) becomes

$$
v_{2}^{4}+e_{33} v_{2}^{3}+e_{32} v_{2}^{2}+e_{31} v_{2}+e_{30}=0
$$

Define

$$
\begin{gathered}
f_{2}\left(v_{2}\right)=v_{2}^{4}+e_{33} v_{2}^{3}+e_{32} v_{2}^{2}+e_{31} v_{2}+e_{30}, \\
m=\frac{1}{2} e_{32}-\frac{3}{16} e_{33}^{2}, \quad n=\frac{1}{32} e_{33}^{3}-\frac{1}{8} e_{32} e_{33}+e_{31}, \\
\alpha_{2}=\left(\frac{n}{2}\right)^{3}+\left(\frac{m}{3}\right)^{3}, \quad \beta_{2}=-\frac{1}{2}+\frac{\sqrt{3}}{2} i \\
z_{1}=\sqrt[3]{-\frac{n}{2}+\sqrt{\alpha_{2}}}+\sqrt[3]{-\frac{n}{2}-\sqrt{\alpha_{2}}} \\
z_{2}=\beta_{2} \sqrt[3]{-\frac{n}{2}+\sqrt{\alpha_{2}}}+\beta_{2}^{2} \sqrt[3]{-\frac{n}{2}-\sqrt{\alpha_{2}}} \\
z_{3}=\beta_{2}^{2} \sqrt[3]{-\frac{n}{2}+\sqrt{\alpha_{2}}}+\beta_{2} \sqrt[3]{-\frac{n}{2}-\sqrt{\alpha_{2}}} \\
v_{2 i}=z_{i}-\frac{3 e_{33}}{4}, \quad i=1,2,3 .
\end{gathered}
$$

Next, we assume that $\left(H_{31}\right)$ : the coefficients in $f_{2}\left(v_{2}\right)$ satisfy one of the following conditions in $\left(\mathrm{a}^{\prime}\right)-\left(\mathrm{c}^{\prime}\right)$ :

(a') $e_{30}<0$;

$\left(\mathrm{b}^{\prime}\right) e_{30} \geq 0, \alpha_{2} \geq 0, v_{21}>0$, and $f_{2}\left(v_{21}\right)<0$;

(c') $e_{30} \geq 0, \alpha_{2}<0$, and there exists at least one $v_{2 *} \epsilon$ $\left\{v_{21}, v_{22}, v_{23}\right\}$ such that $v_{2 *}>0$ and $f_{2}\left(v_{2 *}\right) \leq 0$.

According to Lemma 1, we know that if the condition $\left(H_{31}\right)$ holds, then (28) has at least a positive root $\omega_{20}$ such that (25) has a pair of purely imaginary roots $\pm i \omega_{20}$. The corresponding critical value of the delay is

$$
\begin{aligned}
\tau_{2 k}= & \frac{1}{\omega_{20}} \arccos \frac{m_{6} \omega_{20}^{6}+m_{4} \omega_{20}^{4}+m_{2} \omega_{20}^{2}+m_{0}}{n_{6} \omega_{20}^{6}+n_{4} \omega_{20}^{4}+n_{2} \omega_{20}^{2}+n_{0}} \\
& +\frac{2 k \pi}{\omega_{20}}, \quad k=0,1,2, \ldots,
\end{aligned}
$$

where

$$
\begin{gathered}
m_{0}=-A_{30} C_{30}, \quad m_{2}=A_{30} C_{32}-A_{31} C_{31}+A_{32} C_{30}, \\
m_{4}=A_{31} C_{33}-A_{32} C_{32}+A_{33} C_{31}-C_{30}, \\
m_{6}=C_{32}-A_{33} C_{33}, \quad n_{0}=C_{30}^{2}, \quad n_{2}=C_{31}^{2}-2 C_{30} C_{32}, \\
n_{4}=C_{32}^{2}-2 C_{31} C_{33}, \quad n_{6}=C_{33}^{2} .
\end{gathered}
$$

As in Case 2, we know that if $f_{2}^{\prime}\left(v_{2}^{*}\right) \neq 0$ where $v_{2}^{*}=\omega_{20}^{2}$, then $\operatorname{Re}\left[d \lambda / d \tau_{2}\right]_{\tau_{2}=\tau_{20}}^{-1} \neq 0$. That is, the transversality condition is satisfied if the condition $\left(H_{32}\right) f_{2}^{\prime}\left(v_{2}^{*}\right) \neq 0$ holds. Thus, according to the Hopf bifurcation theorem in [24], we have the following results.

Theorem 3. Suppose the conditions $\left(H_{31}\right)-\left(H_{32}\right)$ hold.

(i) The positive equilibrium $D_{*}\left(S_{*}, E_{*}, I_{*}, R_{*}\right)$ of system (3) is asymptotically stable for $\tau_{2} \in\left[0, \tau_{20}\right)$.

(ii) System (3) undergoes a Hopf bifurcation at the positive equilibrium $D_{*}\left(S_{*}, E_{*}, I_{*}, R_{*}\right)$ when $\tau_{2}=\tau_{20}$ and $a$ family of periodic solutions bifurcate from the positive equilibrium $D_{*}\left(S_{*}, E_{*}, I_{*}, R_{*}\right)$ near $\tau_{2}=\tau_{20}$.

Case $4\left(\tau_{1}=\tau_{2}=\tau>0\right)$. Substituting $\tau_{1}=\tau_{2}=\tau>0$ into (8), then (8) becomes

$$
\begin{aligned}
\lambda^{4}+ & A_{43} \lambda^{3}+A_{42} \lambda^{2}+A_{41} \lambda+A_{40} \\
& +\left(B_{43} \lambda^{3}+B_{42} \lambda^{2}+B_{41} \lambda+B_{40}\right) e^{-\lambda \tau} \\
& +\left(D_{42} \lambda^{2}+D_{41} \lambda+D_{40}\right) e^{-2 \lambda \tau}=0,
\end{aligned}
$$

where

$$
\begin{gathered}
A_{43}=A_{3}, \quad A_{42}=A_{2}, \quad A_{41}=A_{1}, \quad A_{40}=A_{0}, \\
B_{43}=B_{3}+C_{3}, \quad B_{42}=B_{2}+C_{2}, \quad B_{41}=B_{1}+C_{1}, \\
B_{40}=B_{0}+C_{0}, \quad D_{42}=D_{2}, \quad D_{41}=D_{1}, \\
D_{40}=D_{0} .
\end{gathered}
$$

Multiplying by $e^{\lambda \tau},(34)$ becomes

$$
\begin{aligned}
B_{43} \lambda^{3} & +B_{42} \lambda^{2}+B_{41} \lambda+B_{40} \\
& \times\left(\lambda^{4}+A_{43} \lambda^{3}+A_{42} \lambda^{2}+A_{41} \lambda+A_{40}\right) e^{\lambda \tau} \\
& +\left(D_{42} \lambda^{2}+D_{41} \lambda+D_{40}\right) e^{-\lambda \tau}=0 .
\end{aligned}
$$


Let $\lambda=i \omega(\omega>0)$ be the root of (36); consider

$$
\begin{aligned}
\left(\omega^{4}-\right. & \left.\left(A_{42}+D_{42}\right) \omega^{2}+A_{40}+D_{40}\right) \cos \tau \omega \\
& +\left(A_{43} \omega^{3}-\left(A_{41}-D_{41}\right) \omega\right) \sin \tau \omega=B_{32} \omega^{2}-B_{40}, \\
\left(\omega^{4}-\right. & \left.\left(A_{42}-D_{42}\right) \omega^{2}+A_{40}-D_{40}\right) \sin \tau \omega \\
& -\left(A_{43} \omega^{3}-\left(A_{41}+D_{41}\right) \omega\right) \cos \tau \omega=B_{43} \omega^{3}-B_{41} \omega,
\end{aligned}
$$

which follows that

$$
\begin{aligned}
& \sin \tau \omega=\frac{g_{7} \omega^{7}+g_{5} \omega^{5}+g_{3} \omega^{3}+g_{1} \omega}{\omega^{8}+h_{6} \omega^{6}+h_{4} \omega^{4}+h_{2} \omega^{2}+h_{0}}, \\
& \cos \tau \omega=\frac{g_{6} \omega^{6}+g_{4} \omega^{4}+g_{2} \omega^{2}+g_{0}}{\omega^{8}+h_{6} \omega^{6}+h_{4} \omega^{4}+h_{2} \omega^{2}+h_{0}},
\end{aligned}
$$

where

$$
\begin{gathered}
g_{0}=B_{40}\left(D_{40}-A_{40}\right), \\
g_{1}=B_{40}\left(A_{41}+D_{41}\right)-B_{41}\left(A_{40}+D_{40}\right), \\
g_{2}=B_{40}\left(A_{42}-D_{42}\right)-B_{41}\left(A_{41}-D_{41}\right)+B_{42}\left(A_{40}-D_{40}\right), \\
g_{3}=B_{41}\left(A_{42}+D_{42}\right)-B_{42}\left(A_{41}+D_{41}\right) \\
+B_{43}\left(A_{40}+D_{40}\right)-A_{43} B_{40}, \\
g_{4}=B_{42}\left(D_{42}-A_{42}\right)+B_{43}\left(A_{41}-D_{41}\right)+A_{43} B_{41}-B_{40}, \\
g_{5}=A_{42} B_{43}-B_{41}-B_{43}\left(A_{42}+D_{42}\right), \\
g_{6}=B_{42}-A_{43} B_{43}, \\
h_{0}=A_{40}^{2}-D_{40}^{2}, \\
h_{2}=A_{41}^{2}-D_{41}^{2}-2 A_{40} A_{42}+2 D_{40} D_{42}, \\
h_{4}=A_{42}^{2}-D_{42}^{2}-2 A_{41} A_{43}+2 A_{40}, \\
h_{6}=A_{43}^{2}-2 A_{42} .
\end{gathered}
$$

As is known, $\sin ^{2} \tau \omega+\cos ^{2} \tau \omega=1$. Thus, we have

$$
\begin{gathered}
\omega^{16}+e_{47} \omega^{14}+e_{46} \omega^{12}+e_{45} \omega^{10}+e_{44} \omega^{8} \\
+e_{43} \omega^{6}+e_{42} \omega^{4}+e_{41} \omega^{2}+e_{40}=0,
\end{gathered}
$$

where

$$
\begin{gathered}
e_{40}=h_{0}^{2}-g_{0}^{2}, \quad e_{41}=2 h_{0} h_{2}-2 g_{0} g_{2}-g_{1}^{2}, \\
e_{42}=h_{2}^{2}-g_{2}^{2}+2 h_{0} h_{4}-2 g_{0} g_{4}-2 g_{1} g_{3}, \\
e_{43}=2 h_{0} h_{6}+2 h_{2} h_{4}-2 g_{0} g_{6}-2 g_{1} g_{5}-2 g_{2} g_{4}-g_{3}^{3}, \\
e_{44}=h_{4}^{2}-g_{4}^{2}+2 h_{0}+2 h_{2} h_{6}-2 g_{1} g_{7}-2 g_{2} g_{6}-2 g_{3} g_{5}, \\
e_{45}=2 h_{2}+2 h_{4} h_{6}-2 g_{3} g_{7}-2 g_{4} g_{6}-g_{5}^{2},
\end{gathered}
$$

$$
\begin{gathered}
e_{46}=h_{6}^{2}-g_{6}^{2}+2 h_{4}-2 g_{5} g_{7}, \\
e_{47}=2 h_{6}-g_{7}^{2} .
\end{gathered}
$$

Let $\omega^{2}=v$, then (40) becomes

$$
\begin{gathered}
v^{8}+e_{47} v^{7}+e_{46} v^{6}+e_{45} v^{5}+e_{44} v^{4}+e_{43} v^{3} \\
+e_{42} v^{2}+e_{41} v+e_{40}=0 .
\end{gathered}
$$

In order to give the main results in this paper, we make the following assumption.

$\left(H_{41}\right)(42)$ has at least one positive real root.

Suppose that condition $\left(H_{41}\right)$ holds. Without loss of generality, we assume that (42) has eight positive real roots, which are denoted as $v_{1}, v_{2}, \ldots, v_{8}$, respectively. Then (40) has eight positive real roots $\omega_{k}=\sqrt{v_{k}}$. For every fixed $\omega_{k}$, the corresponding critical value of time delay is

$$
\begin{array}{r}
\tau_{k}^{(j)}=\frac{1}{\omega_{k}} \arccos \frac{g_{6} \omega_{k}^{6}+g_{4} \omega_{k}^{4}+g_{2} \omega_{k}^{2}+g_{0}}{\omega_{k}^{8}+h_{6} \omega_{k}^{6}+h_{4} \omega_{k}^{4}+h_{2} \omega_{k}^{2}+h_{0}}+\frac{2 j \pi}{\omega_{k}}, \\
k=1,2, \ldots ; \quad j=0,1,2, \ldots
\end{array}
$$

Define

$$
\tau_{0}=\min \left\{\tau_{k}^{(0)} \mid k=1,2, \ldots, 8\right\}, \quad \omega_{0}=\left.\omega_{k}\right|_{\tau=\tau_{0}} .
$$

Taking the derivative of with respect to (36), it is easy to obtain

$$
\begin{aligned}
& {\left[\frac{d \lambda}{d \tau}\right]^{-1}} \\
& =-\left(\left(3 B_{43} \lambda^{2}+2 B_{42} \lambda+B_{41}+\left(2 D_{42} \lambda+D_{41}\right) e^{-\lambda \tau}\right.\right. \\
& \left.+\left(4 \lambda^{3}+3 A_{43} \lambda^{2}+2 A_{42} \lambda+A_{41}\right) e^{\lambda \tau}\right) \\
& \quad \times\left(\left(\lambda^{5}+A_{43} \lambda^{4}+A_{42} \lambda^{3}+A_{41} \lambda^{2}+A_{40} \lambda\right) e^{\lambda \tau}\right. \\
& \left.\left.\quad-\left(D_{42} \lambda^{3}+D_{41} \lambda^{2}+D_{40} \lambda\right) e^{-\lambda \tau}\right)^{-1}\right)-\frac{\tau}{\lambda} .
\end{aligned}
$$

Thus, we have

$$
\operatorname{Re}\left[\frac{d \lambda}{d \tau}\right]_{\tau=\tau_{0}}^{-1}=-\frac{P_{R} Q_{R}+P_{I} Q_{I}}{Q_{R}^{2}+Q_{I}^{2}},
$$

where

$$
\begin{aligned}
P_{R}= & \left(A_{41}+D_{41}-3 A_{43} \omega_{0}^{2}\right) \cos \tau_{0} \omega_{0} \\
& +2\left(2 \omega_{0}^{3}-A_{42} \omega_{0}+D_{42} \omega_{0}\right) \sin \tau_{0} \omega_{0}-3 B_{43} \omega_{0}^{2}+B_{41}, \\
P_{I}= & \left(A_{41}-D_{41}-3 A_{43} \omega_{0}^{2}\right) \sin \tau_{0} \omega_{0} \\
& -2\left(2 \omega_{0}^{3}-A_{42} \omega_{0}-D_{42} \omega_{0}\right) \cos \tau_{0} \omega_{0}+2 B_{42} \omega_{0} \\
Q_{R}= & \left(\omega_{0}^{5}-\left(A_{42}+D_{42}\right) \omega_{0}^{3}+\left(A_{40}+D_{40}\right) \omega_{0}\right) \sin \tau_{0} \omega_{0} \\
& +\left(A_{43} \omega_{0}^{4}-\left(A_{41}+D_{41}\right) \omega_{0}^{2}\right) \cos \tau_{0} \omega_{0}
\end{aligned}
$$




$$
\begin{aligned}
Q_{I}= & \left(\omega_{0}^{5}-\left(A_{42}+D_{42}\right) \omega_{0}^{3}+\left(A_{40}+D_{40}\right) \omega_{0}\right) \cos \tau_{0} \omega_{0} \\
& -\left(A_{43} \omega_{0}^{4}-\left(A_{41}+D_{41}\right) \omega_{0}^{2}\right) \sin \tau_{0} \omega_{0} .
\end{aligned}
$$

Obviously, if condition $P_{R} Q_{R}+P_{I} Q_{I} \neq 0$ holds, then $\operatorname{Re}[d \lambda / d \tau]_{\tau=\tau_{0}}^{-1} \neq 0$. Thus, by the Hopf bifurcation theorem in [24], we have the following results.

Theorem 4. Suppose that conditions $\left(H_{41}\right)-\left(H_{42}\right)$ hold.

(i) The positive equilibrium $D_{*}\left(S_{*}, E_{*}, I_{*}, R_{*}\right)$ of system (3) is asymptotically stable for $\tau \in\left[0, \tau_{0}\right)$.

(ii) System (3) undergoes a Hopf bifurcation at the positive equilibrium $D_{*}\left(S_{*}, E_{*}, I_{*}, R_{*}\right)$ when $\tau=\tau_{0}$ and $a$ family of periodic solutions bifurcate from the positive equilibrium $D_{*}\left(S_{*}, E_{*}, I_{*}, R_{*}\right)$ near $\tau=\tau_{0}$.

Case $5\left(\tau_{1}>0, \tau_{2} \in\left(0, \tau_{20}\right)\right)$. We consider (8) with $\tau_{2}$ in its stable interval, regarding $\tau_{1}$ as a parameter.

Let $\lambda=i \omega_{1 *}\left(\omega_{1 *}>0\right)$ be the root of $(8)$; we obtain

$c_{0}\left(\omega_{1 *}\right)+2 c_{1}\left(\omega_{1 *}\right) \cos \tau_{2} \omega_{1 *}+2 c_{2}\left(\omega_{1 *}\right) \sin \tau_{2} \omega_{1 *}=0$,

where

$$
\begin{aligned}
c_{0}\left(\omega_{1 *}\right)= & \omega_{1 *}^{8}+\left(A_{3}^{2}-B_{3}^{2}+C_{3}^{2}-2 A_{2}\right) \omega_{1 *}^{6} \\
& +\left(A_{2}^{2}-B_{2}^{2}+C_{2}^{2}-D_{2}^{2}+2 A_{0}-2 A_{1} A_{3}+2 B_{1} B_{3}\right. \\
& \left.\quad-2 C_{1} C_{3}\right) \omega_{1 *}^{4} \\
& +\left(A_{1}^{2}-B_{1}^{2}+C_{1}^{2}-D_{1}^{2}-2 A-0 A_{2}\right. \\
& \left.\quad+2 B_{0} B_{2}-2 C_{0} C_{2}+2 D_{0} D_{2}\right) \omega_{1 *}^{2} \\
& +A_{0}^{2}-B_{0}^{2}+C_{0}^{2}-D_{0}^{2}, \\
c_{1}\left(\omega_{1 *}\right)= & \left(A_{3} C_{3}-C_{2}\right) \omega_{1 *}^{6} \\
& +\left(A_{2} C_{2}+B_{3} D_{1}-B_{2} D_{2}+C_{0}-A_{1} C_{3}-A_{3} C_{1}\right) \\
& \times \omega_{1 *}^{4}+\left(A_{1} C_{1}-A_{0} C_{2}-A_{2} C_{0}+B_{0} D_{2}\right. \\
& \left.\quad+B_{2} D_{0}-B_{1} D_{1}\right) \omega_{1 *}^{2}+A_{0} C_{0}+B_{0} D_{0} \\
& +\left(A_{0} C_{1}-A_{1} C_{0}-B_{0} D_{1}+B_{1} D_{0}\right) \omega_{1 *} \cdot \\
& \left.+B_{2} D_{1}-B_{3} D_{0}\right) \omega_{1 *}^{3} \\
& +A_{3} C_{0}-A_{0} C_{3}-A_{2} C_{1}-B_{1} D_{2} \\
& +C_{3} \omega_{1 *}^{7}+\left(A_{2} C_{3}-A_{3} C_{2}+B_{3} D_{2}+C_{1}\right) \omega_{1 *}^{5} \\
& \left(A_{1} C_{2}\right)
\end{aligned}
$$

We assume that $\left(H_{51}\right)(48)$ has at least finite positive roots. And we denote the positive roots of $(48)$ as $\omega_{11 *}, \omega_{12 *}$, and $\omega_{1 k *}$. For every fixed $\omega_{1 i *}$, the corresponding critical value of time delay is

$$
\begin{array}{r}
\tau_{1 i *}^{(j)}=\frac{1}{\omega_{1 i *}} \arccos \frac{\Delta_{1} \Delta_{4}+\Delta_{2} \Delta_{3}}{\Delta_{1}^{2}+\Delta_{2}^{2}}+\frac{2 j \pi}{\omega_{1 i *}}, \\
i=1,2, k ; \quad j=0,1,2, \ldots,
\end{array}
$$

where

$$
\begin{aligned}
\Delta_{1}= & D_{1} \omega_{1 i *} \cos \tau_{2} \omega_{1 i *}+\left(D_{2} \omega_{1 i *}^{2}-D_{0}\right) \sin \tau_{2} \omega_{1 i *} \\
& -B_{3} \omega_{1 i *}^{3}+B_{1} \omega_{1 i *}, \\
\Delta_{2}= & D_{1} \omega_{1 i *} \sin \tau_{2} \omega_{1 i *}-\left(D_{2} \omega_{1 i *}^{2}-D_{0}\right) \cos \tau_{2} \omega_{1 i *} \\
& -B_{2} \omega_{1 i *}^{2}+B_{0}, \\
\Delta_{3}= & \left(C_{3} \omega_{1 i *}^{3}-C_{1} \omega_{1 i *}\right) \sin \tau_{2} \omega_{1 i *} \\
& +\left(C_{2} \omega_{1 i *}^{2}-C_{0}\right) \cos \tau_{2} \omega_{1 i *}+A_{2} \omega_{1 i *}^{2}-\omega_{1 i *}^{4}-A_{0}, \\
\Delta_{4}= & \left(C_{3} \omega_{1 i *}^{3}-C_{1} \omega_{1 i *}\right) \cos \tau_{2} \omega_{1 i *} \\
& +\left(C_{2} \omega_{1 i *}^{2}-C_{0}\right) \sin \tau_{2} \omega_{1 i *}+A_{3} \omega_{1 i *}^{3}-A_{1} \omega_{1 i *} .
\end{aligned}
$$

Let

$$
\tau_{1}^{*}=\min \left\{\tau_{1 i *}^{(0)} \mid i=1,2, \ldots, k\right\}, \quad \omega_{1}^{*}=\left.\omega_{1 i *}\right|_{\tau_{1}=\tau_{1}^{*}} .
$$

Next, we make the following assumption: $\left(H_{51}\right) \operatorname{Re}[d \lambda /$ $d \tau]_{\tau_{1}=\tau_{1}^{*}}^{-1} \neq 0$. Thus, by the Hopf bifurcation theorem in [24], we have the following results.

Theorem 5. Suppose that conditions $\left(H_{51}\right)-\left(H_{52}\right)$ hold and $\tau_{2} \in\left(0, \tau_{20}\right)$.

(i) The positive equilibrium $D_{*}\left(S_{*}, E_{*}, I_{*}, R_{*}\right)$ of system (3) is asymptotically stable for $\tau_{1} \in\left[0, \tau_{1}^{*}\right)$.

(ii) System (3) undergoes a Hopf bifurcation at the positive equilibrium $D_{*}\left(S_{*}, E_{*}, I_{*}, R_{*}\right)$ when $\tau_{1}=\tau_{1}^{*}$ and $a$ family of periodic solutions bifurcate from the positive equilibrium $D_{*}\left(S_{*}, E_{*}, I_{*}, R_{*}\right)$ near $\tau_{1}=\tau_{1}^{*}$.

\section{Direction and Stability of the Hopf Bifurcation}

In this section, we will investigate the direction of the Hopf bifurcation and the stability of the bifurcating periodic solutions w.r.t. $\tau_{1}$ for $\tau_{2} \in\left(0, \tau_{20}\right)$ by using the normal form theory and the center manifold argument in [24]. We assume that $\tau_{2}^{*}<\tau_{1}^{*}$ where $\tau_{2}^{*} \in\left(0, \tau_{20}\right)$.

Let $u_{1}(t)=S(t)-S_{*}, u_{2}(t)=E(t)-E_{*}, u_{3}(t)=I(t)-I_{*}$, $u_{4}(t)=R(t)-R_{*}, \tau_{1}=\tau_{1}^{*}+\mu, \mu \in R$, and normalize $t \rightarrow$ $\left(t / \tau_{1}\right)$. Then system (3) can be transformed into the following form:

$$
\dot{u}(t)=L_{\mu} u_{t}+F\left(\mu, u_{t}\right),
$$

where $u_{t}=\left(u_{1}(t), u_{2}(t), u_{3}(t), u_{4}(t)\right)^{T} \in C=C\left([-1,0], R^{4}\right)$ and

$$
\begin{gathered}
L_{\mu} \phi=\left(\tau_{1}^{*}+\mu\right)\left(A^{\prime} \phi(0)+C^{\prime} \phi\left(-\frac{\tau_{2}^{*}}{\tau_{1}^{*}}\right)+B^{\prime} \phi(-1)\right), \\
F(\mu, \phi)=\left(\tau_{1}^{*}+\mu\right)\left(\begin{array}{c}
-\beta \phi_{1}(0) \phi_{3}(0) \\
\beta \phi_{1}(0) \phi_{3}(0) \\
0 \\
0
\end{array}\right),
\end{gathered}
$$


where

$$
\begin{aligned}
A^{\prime} & =\left(\begin{array}{cccc}
a_{11} & 0 & a_{13} & 0 \\
a_{21} & a_{22} & a_{23} & 0 \\
0 & a_{32} & a_{33} & 0 \\
a_{41} & 0 & 0 & a_{44}
\end{array}\right) \\
B^{\prime} & =\left(\begin{array}{cccc}
0 & 0 & 0 & 0 \\
0 & b_{22} & 0 & 0 \\
0 & 0 & 0 & 0 \\
0 & b_{42} & b_{43} & 0
\end{array}\right) \\
C^{\prime} & =\left(\begin{array}{cccc}
0 & 0 & 0 & c_{14} \\
0 & 0 & 0 & 0 \\
0 & 0 & 0 & 0 \\
0 & 0 & 0 & c_{44}
\end{array}\right)
\end{aligned}
$$

By the Riesz representation theorem, there exists a $4 \times 4$ matrix function $\eta(\theta, \mu):[-1,0] \rightarrow R^{4}$ whose elements are of bounded variation such that

$$
L_{\mu} \phi=\int_{-1}^{0} d \eta(\theta, \mu) \phi(\theta), \quad \phi \in C .
$$

In fact, we choose

$$
\eta(\theta, \mu)= \begin{cases}\left(\tau_{1}^{*}+\mu\right)\left(A^{\prime}+B^{\prime}+C^{\prime}\right), & \theta=0, \\ \left(\tau_{1}^{*}+\mu\right)\left(B^{\prime}+C^{\prime}\right), & \theta \in\left[-\frac{\tau_{2}^{*}}{\tau_{1}^{*}}, 0\right), \\ \left(\tau_{1}^{*}+\mu\right) B^{\prime}, & \theta \in\left(-1,-\frac{\tau_{2}^{*}}{\tau_{1}^{*}}\right), \\ 0, & \theta=1 .\end{cases}
$$

For $\phi \in C\left([-1,0], R^{4}\right)$, we define

$$
\begin{gathered}
A(\mu) \phi= \begin{cases}\frac{d \phi(\theta)}{d \theta}, & -1 \leq \theta<0, \\
\int_{-1}^{0} d \eta(\theta, \mu) \phi(\theta), & \theta=0,\end{cases} \\
R(\mu) \phi= \begin{cases}0, & -1 \leq \theta<0, \\
F(\mu, \phi), & \theta=0 .\end{cases}
\end{gathered}
$$

Then system (53) can be transformed into the following operator equation:

$$
\dot{u}(t)=A(\mu) u_{t}+R(\mu) u_{t} .
$$

The adjoint operator $A^{*}$ of $A$ is defined by

$$
A^{*}(\varphi)= \begin{cases}-\frac{d \varphi(s)}{d s}, & 0<s \leq 1, \\ \int_{-1}^{0} d \eta^{T}(s, 0) \varphi(-s), & s=0,\end{cases}
$$

associated with a bilinear form

$$
\begin{aligned}
\langle\varphi(s), \phi(\theta)\rangle= & \bar{\varphi}(0) \phi(0) \\
& -\int_{\theta=-1}^{0} \int_{\xi=0}^{\theta} \bar{\varphi}(\xi-\theta) d \eta(\theta) \phi(\xi) d \xi,
\end{aligned}
$$

where $\eta(\theta)=\eta(\theta, 0)$.
Let $q(\theta)=\left(1, q_{2}, q_{3}, q_{4}\right)^{T} e^{i \tau_{1}^{*} \omega_{1}^{*} \theta}$ be the eigenvector of $A(0)$ corresponding to $+i \tau_{1}^{*} \omega_{1}^{*}$, and let $q^{*}(s)=D\left(1, q_{2}^{*}, q_{3}^{*}\right.$, $\left.q_{4}^{*}\right) e^{i \tau_{1}^{*} \omega_{1}^{*} s}$ be the eigenvector of $A^{*}(0)$ corresponding to $-i \tau_{1}^{*} \omega_{1}^{*}$. From the definition of $A(0)$ and $A^{*}(0)$, we can get

$$
\begin{aligned}
& q_{2}=\frac{a_{21}\left(i \omega_{1}^{*}-a_{33}\right)}{\left(i \omega_{1}^{*}-a_{33}\right)\left(i \omega_{1}^{*}-a_{22}-b_{22} e^{-\tau_{1}^{*} \omega_{1}^{*}}\right)-a_{23} a_{32}}, \\
& q_{3}=\frac{a_{21} a_{32}}{\left(i \omega_{1}^{*}-a_{33}\right)\left(i \omega_{1}^{*}-a_{22}-b_{22} e^{-\tau_{1}^{*} \omega_{1}^{*}}\right)-a_{23} a_{32}}, \\
& q_{4}=\frac{i \omega_{1}^{*}-a_{11}}{c_{14} e^{-i \tau_{2}^{*} \omega_{1}^{*}}} \\
& -\frac{a_{13} a_{21} a_{32} e^{i \tau_{2}^{*} \omega_{1}^{*}}}{c_{14}\left(i \omega_{1}^{*}-a_{33}\right)\left(i \omega_{1}^{*}-a_{22}-b_{22} e^{-\tau_{1}^{*} \omega_{1}^{*}}\right)-a_{23} a_{32} c_{14}}, \\
& q_{2}^{*}=\frac{a_{41} c_{14} e^{i \tau_{2}^{*} \omega_{1}^{*}}}{a_{21}\left(i \omega_{1}^{*}+a_{44}+c_{44} e^{i \tau_{2}^{*} \omega_{1}^{*}}\right)}-\frac{i \omega_{1}^{*}+a_{11}}{a_{21}}, \\
& q_{3}^{*}=-\frac{\left(i \omega_{1}^{*}+a_{22}+b_{22} e^{i \tau_{1}^{*} \omega_{1}^{*}}\right) q_{2}^{*}+b_{42} e^{i \tau_{1}^{*} \omega_{1}^{*}} q_{4}^{*}}{a_{32}}, \\
& q_{4}^{*}=-\frac{c_{14} e^{i \tau_{2}^{*} \omega_{1}^{*}}}{i \omega_{1}^{*}+a_{44}+c_{44} e^{i \tau_{2}^{*} \omega_{1}^{*}}} . \\
& \text { From (61), we can get } \\
& \left\langle q^{*}, q\right\rangle=\bar{D}\left[1+q_{2} \bar{q}_{2}^{*}+q_{3} \bar{q}_{3}^{*}+q_{4} \bar{q}_{4}^{*}\right. \\
& +\tau_{2}^{*} e^{-i \tau_{2}^{*} \omega_{1}^{*}} q_{4}\left(c_{14}+c_{44} \bar{q}_{4}^{*}\right) \\
& \left.+\tau_{1}^{*} e^{-i \tau_{1}^{*} \omega_{1}^{*}}\left(q_{2}\left(b_{22} \bar{q}_{2}^{*}+b_{42} \bar{q}_{4}^{*}\right)+b_{43} q_{3} \bar{q}_{4}^{*}\right)\right] .
\end{aligned}
$$

Then, we choose

$$
\begin{gathered}
\bar{D}=\left[1+q_{2} \bar{q}_{2}^{*}+q_{3} \bar{q}_{3}^{*}+q_{4} \bar{q}_{4}^{*}+\tau_{2}^{*} e^{-i \tau_{2}^{*} \omega_{1}^{*}} q_{4}\left(c_{14}+c_{44} \bar{q}_{4}^{*}\right)\right. \\
\left.+\tau_{1}^{*} e^{-i \tau_{1}^{*} \omega_{1}^{*}}\left(q_{2}\left(b_{22} \bar{q}_{2}^{*}+b_{42} \bar{q}_{4}^{*}\right)+b_{43} q_{3} \bar{q}_{4}^{*}\right)\right]^{-1},
\end{gathered}
$$

such that $\left\langle q^{*}, q\right\rangle=1,\left\langle q^{*}, \bar{q}\right\rangle=0$.

Next, we can get the coefficients which can be used to determine the direction of the Hopf bifurcation and the stability of the bifurcating periodic solutions by following the algorithms introduced in [24]:

$$
\begin{gathered}
g_{20}=2 \beta \tau_{1}^{*} \bar{D} q_{3}\left(\bar{q}_{2}^{*}-1\right), \\
g_{02}=2 \beta \tau_{1}^{*} \bar{D} \bar{q}_{3}\left(\bar{q}_{2}^{*}-1\right), \\
g_{11}=b t_{1}^{*} \bar{D}\left(q_{3}+\bar{q}_{3}\right)\left(\bar{q}_{2}^{*}-1\right), \\
g_{21}=2 \beta \tau_{1}^{*} \bar{D}\left(\bar{q}_{2}^{*}-1\right)\left(W_{11}^{(1)}(0) q_{3}+\frac{1}{2} W_{20}^{(1)}(0) \bar{q}_{3}\right. \\
\left.+W_{11}^{(3)}(0)+\frac{1}{2} W_{20}^{(3)}(0)\right),
\end{gathered}
$$


with

$$
W_{11}(\theta)=-\frac{i g_{11} q(0)}{\tau_{1}^{*} \omega_{1}^{*}} e^{i \tau_{1}^{*} \omega_{1}^{*} \theta}+\frac{i \bar{g}_{11} \bar{q}(0)}{\tau_{1}^{*} \omega_{1}^{*}} e^{-i \tau_{1}^{*} \omega_{1}^{*} \theta}+E_{2},
$$

$$
W_{20}(\theta)=\frac{i g_{20} q(0)}{\tau_{1}^{*} \omega_{1}^{*}} e^{i \tau_{1}^{*} \omega_{1}^{*} \theta}+\frac{i \bar{g}_{02} \bar{q}(0)}{3 \tau_{1}^{*} \omega_{1}^{*}} e^{-i \tau_{1}^{*} \omega_{1}^{*} \theta}+E_{1} e^{2 i \tau_{1}^{*} \omega_{1}^{*} \theta},
$$

where $E_{1}$ and $E_{2}$ can be determined by the following equations respectively

$$
\begin{gathered}
E_{1}=2\left(\begin{array}{cccc}
2 i \omega_{1}^{*}-a_{11} & 0 & -a_{13} & -c_{14} e^{-2 i \tau_{2}^{*} \omega_{1}^{*}} \\
-a_{21} & 2 i \omega_{1}^{*}-a_{22}-b_{22} e^{-2 i \tau_{1}^{*} \omega_{1}^{*}} & -a_{23} & 0 \\
0 & -a_{32} & 2 i \omega_{1}^{*}-a_{33} & 0 \\
-a_{41} & -b_{42} e^{-2 i \tau_{1}^{*} \omega_{1}^{*}} & -b_{43} e^{-2 i \tau_{1}^{*} \omega_{1}^{*}} & 2 i \omega_{1}^{*}-a_{44}-c_{44} e^{-2 i \tau_{2}^{*} \omega_{1}^{*}}
\end{array}\right)^{-1}\left(\begin{array}{c}
E_{1}^{(1)} \\
E_{1}^{(2)} \\
0 \\
0
\end{array}\right), \\
E_{2}=-\left(\begin{array}{cccc}
a_{11} & 0 & a_{13} & c_{14} \\
a_{21} & a_{22}+b_{22} & a_{23} & 0 \\
0 & a_{32} & a_{33} & 0 \\
a_{41} & b_{42} & b_{43} & a_{44}+c_{44}
\end{array}\right)^{-1}\left(\begin{array}{c}
E_{2}^{(1)} \\
E_{2}^{(2)} \\
0 \\
0
\end{array}\right),
\end{gathered}
$$

with

$$
\begin{array}{cl}
E_{1}^{(1)}=-\beta q_{3}, & E_{1}^{(2)}=\beta q_{3}, \\
E_{2}^{(1)}=-\beta\left(q_{3}+\bar{q}_{3}\right), & E_{2}^{(2)}=\beta\left(q_{3}+\bar{q}_{3}\right) .
\end{array}
$$

Therefore, we can calculate the following values:

$$
\begin{gathered}
C_{1}(0)=\frac{i}{2 \tau_{1}^{*} \omega_{1}^{*}}\left(g_{11} g_{20}-2\left|g_{11}\right|^{2}-\frac{\left|g_{02}\right|^{2}}{3}\right)+\frac{g_{21}}{2}, \\
\bar{\mu}=-\frac{\operatorname{Re}\left\{C_{1}(0)\right\}}{\operatorname{Re}\left\{\lambda^{\prime}\left(\tau_{1}^{*}\right)\right\}} \\
\bar{\beta}=2 \operatorname{Re}\left\{C_{1}(0)\right\} \\
\bar{T}=-\frac{\operatorname{Im}\left\{C_{1}(0)\right\}+\bar{\mu} \operatorname{Im}\left\{\lambda^{\prime}\left(\tau_{1}^{*}\right)\right\}}{\tau_{1}^{*} \omega_{1}^{*}} .
\end{gathered}
$$

Based on the discussion above, we can obtain the following results.

Theorem 6. For system (3), if $\bar{\mu}>0(\bar{\mu}<0)$, the Hopf bifurcation is supercritical (subcritical). If $\bar{\beta}<0(\bar{\beta}>0)$ the bifurcating periodic solutions are stable (unstable). If $\bar{T}>0(\bar{T}<$ 0 ), the period of the bifurcating periodic solutions increases (decreases).

\section{Numerical Simulation}

In this section, we present some numerical simulations to verify the theoretical analysis in Sections 2 and 3. Let $N=$ $1000, \alpha=0.1, \beta=0.01, \gamma=0.08, \varepsilon=0.15, \mu=0.01$,
$\rho_{S R}=0.2, \rho_{E R}=0.2$. Then, we can get a particular case of system (3):

$$
\begin{gathered}
\frac{d S(t)}{d t}=10-0.01 I(t) S(t)-0.21 S(t)+0.15 R\left(t-\tau_{2}\right), \\
\frac{d E(t)}{d t}=0.01 I(t) S(t)-0.11 E(t)-0.2 E\left(t-\tau_{1}\right), \\
\quad \frac{d I(t)}{d t}=0.1 E(t)-0.09 I(t), \\
\frac{d R(t)}{d t}=0.2 S(t)+0.2 E\left(t-\tau_{1}\right)+0.08 I\left(t-\tau_{1}\right) \\
-0.01 R(t)-0.15 R\left(t-\tau_{2}\right) .
\end{gathered}
$$

Then, we can get $R_{0}=2.5996>1$ and the unique positive equilibrium $D_{*}(27.9,239.2915,265.8794,466.9290)$

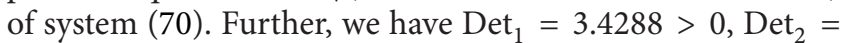
$5.4964>0$, Det $_{3}=0.6898>0$, Det $_{4}=9.6572 e-004>0$. That is, condition $\left(H_{1}\right)$ holds.

For $\tau_{1}>0, \tau_{2}=0$, by some complicated computation, we obtain $\omega_{10}=0.6102, \tau_{10}=17.2530$. By Theorem 2, we know that when $\tau_{1} \in[0,17.2530)$ the positive equilibrium $D_{*}$ is asymptotically stable which can be illustrated by Figures 1 and 2. However, if we let $\tau_{1}=20.05>17.2530=\tau_{10}$, the positive equilibrium $D_{*}$ becomes unstable and a Hopf bifurcation occurs and a branch of periodic solutions bifurcates from the positive equilibrium $D_{*}$. This property can be shown in Figures 3 and 4. Similarly, we have $\omega_{20}=0.1181, \tau_{20}=$ 52.4927 for $\tau_{1}=0, \tau_{2}>0$. The corresponding wave forms and plots are shown in Figures 5, 6, 7, and 8. 


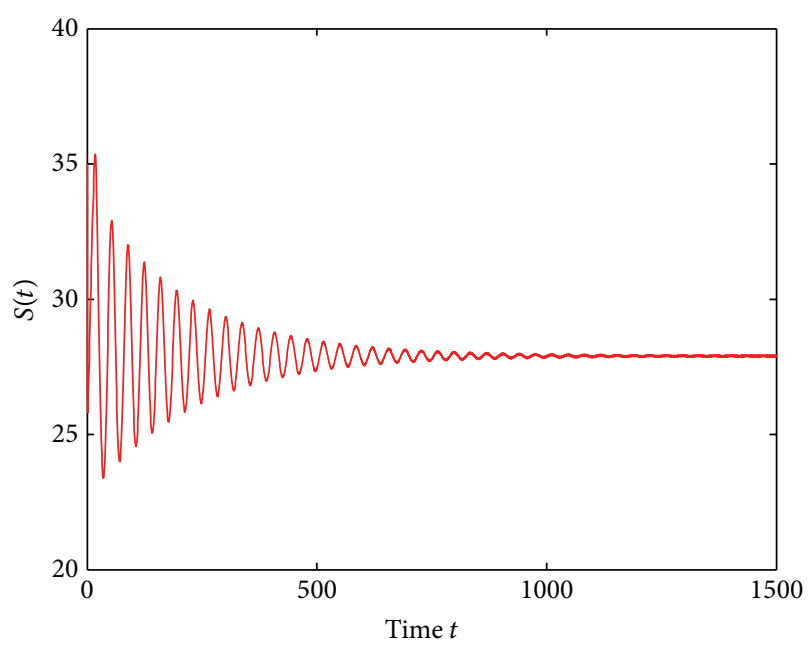

(a)

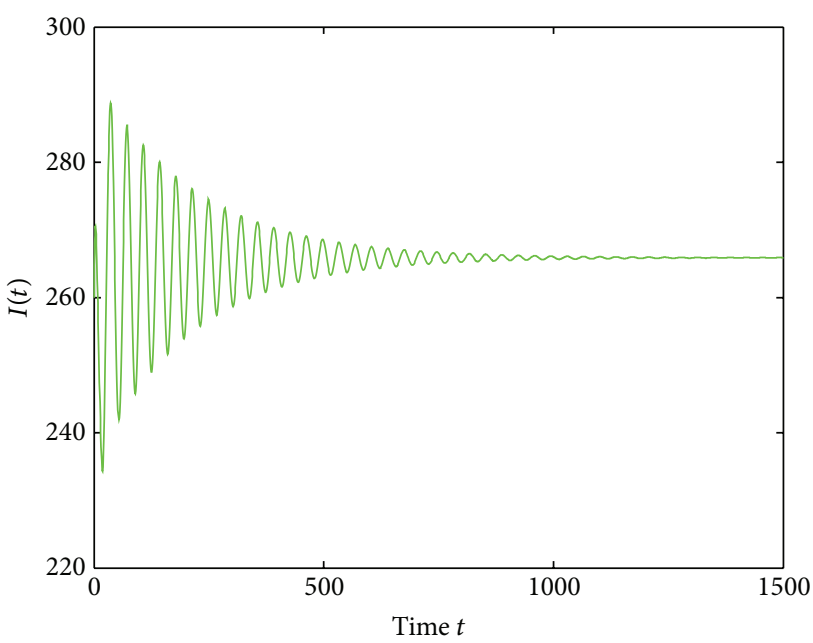

(c)

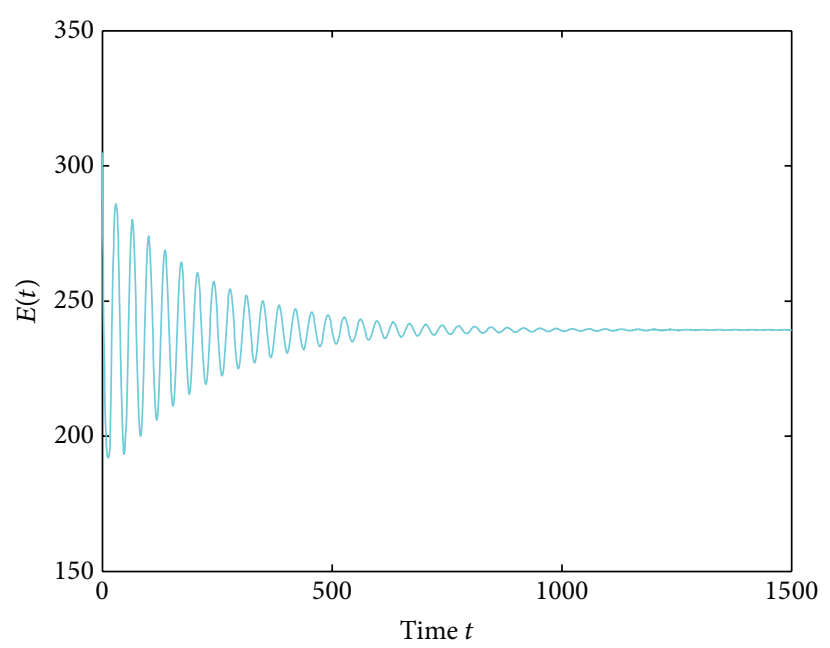

(b)

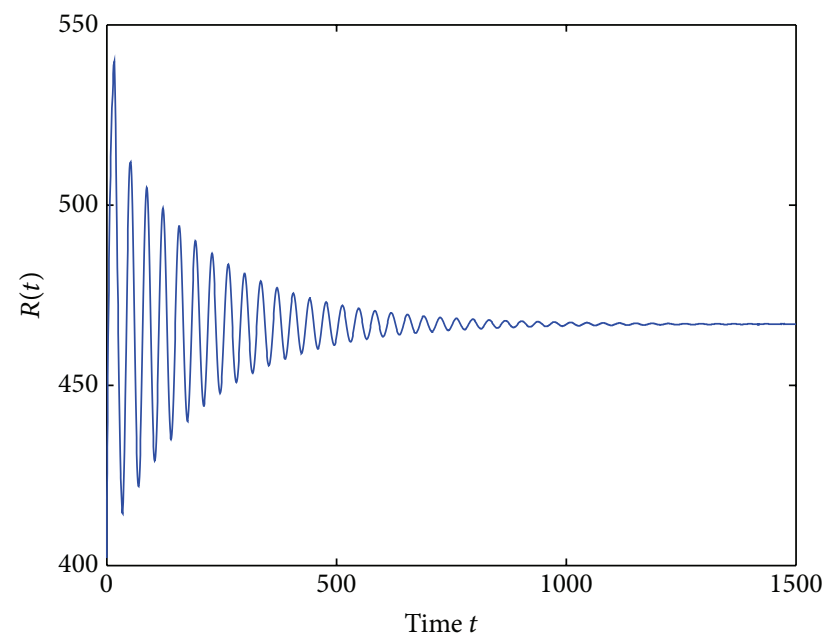

(d)

FIgURE 1: The track of the states $S, E, I$, and $R$ for $\tau_{1}=15.5000<17.2530=\tau_{10}$.

For $\tau_{1}=\tau_{2}=\tau>0$, we get $\omega_{0}=2.0670, \tau_{0}=5.1094$. According to Theorem 4, we can conclude that when $\tau \in$ $[0,5.1094)$, the positive equilibrium $D_{*}$ is asymptotically stable, which can be seen from Figures 9 and 10. If $\tau=5.23>$ $5.1094=\tau_{0}$; then, the positive equilibrium $D_{*}$ becomes unstable, and a branch of bifurcating periodic solutions occurs, which can be shown in Figures 11 and 12.

Lastly, we have $\omega_{1}^{*}=1.6115, \tau_{1}^{*}=7.8533$ for $\tau_{1}>0$, $\tau_{2}=3 \in\left(0, \tau_{20}\right)$. The corresponding wave forms and phase plots are shown in Figures 13, 14, 15, and 16. In addition, we have $\lambda^{\prime}\left(\tau_{1}^{*}\right)=0.3938+0.1113 i, C_{1}(0)=-0.2079-0.6067 i$, $\bar{\mu}=0.5279, \bar{\beta}=-0.4158<0, \bar{T}=0.0433>0$. Thus, by Theorem 6, we can conclude that the Hopf bifurcation is supercritical, the bifurcating periodic solutions are stable, and the period of the periodic solutions increases.

\section{Conclusions}

Based on the SEIR model considered in the literature [10], we propose an SEIRS model with two delays for the propagation of computer viruses in networks. The effects of the two delays on the dynamics of the model are investigated. It is found that the two delays may lead to local Hopf bifurcation and make the model unstable under some certain conditions. When the corresponding delay is suitable and small, the positive equilibrium is asymptotically stable. In this case, the propagation of computer viruses can be predicted and controlled. However, a local Hopf bifurcation occurs and a branch of periodic solutions bifurcates from the positive equilibrium when the corresponding delay passes though a critical value. In such conditions, propagation of the computer viruses is out of control. This phenomenon is not welcome in networks. Therefore, In order to control and even eliminate the propagation of computer viruses, the two delays $\tau_{1}$ and $\tau_{2}$ in the model should remain less than the corresponding critical value. Furthermore, by using the normal form theory and center manifold theorem, the properties of the Hopf bifurcation such as direction and stability are determined. Some numerical simulations are also included to testify the theoretical analysis. 


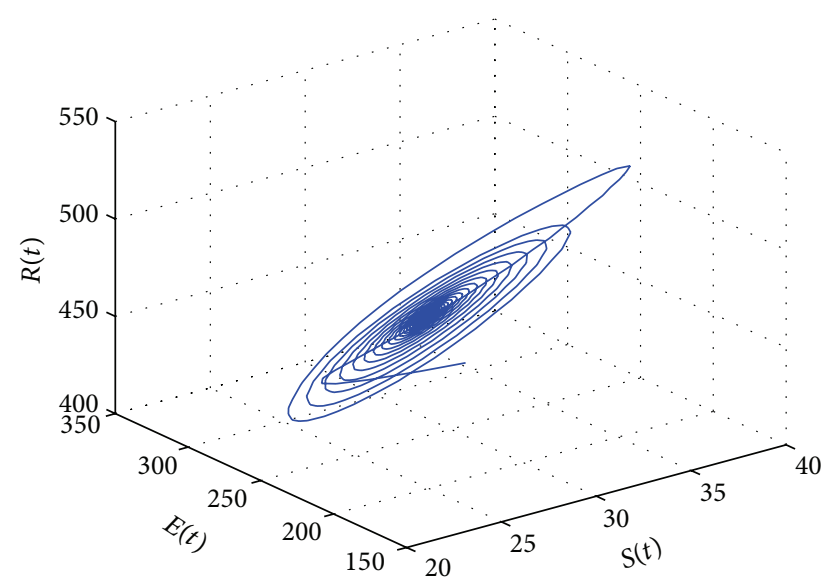

Figure 2: The phase plot of the states $S, E$, and $R$ for $\tau_{1}=15.5000<17.2530=\tau_{10}$.

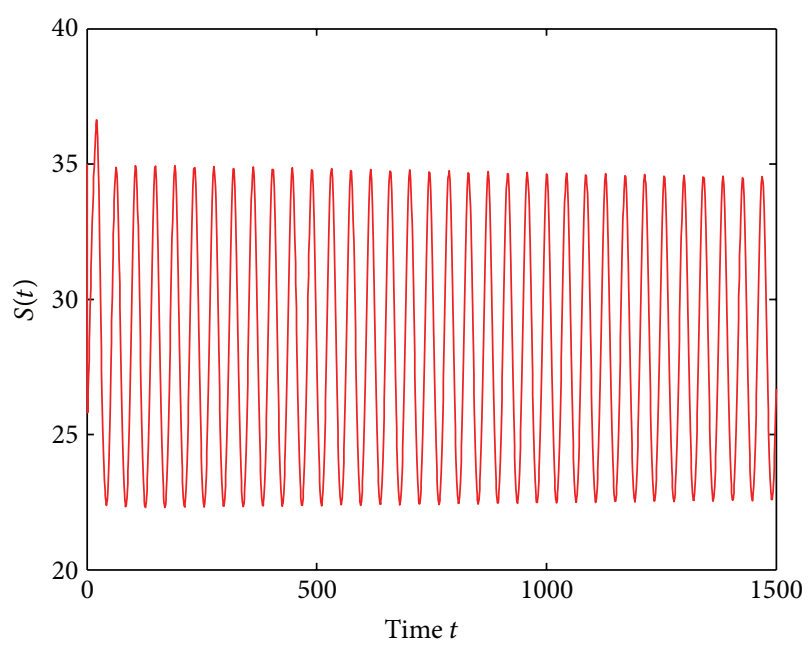

(a)

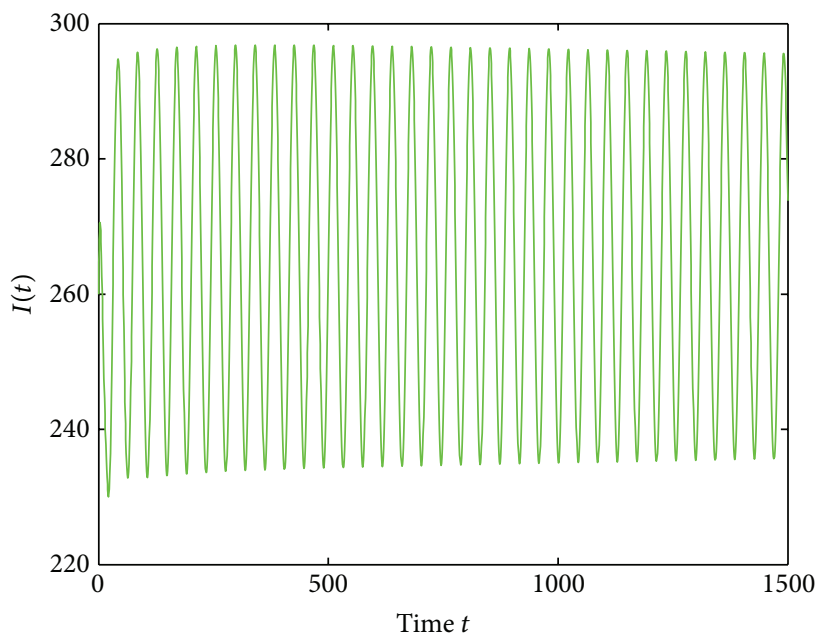

(c)

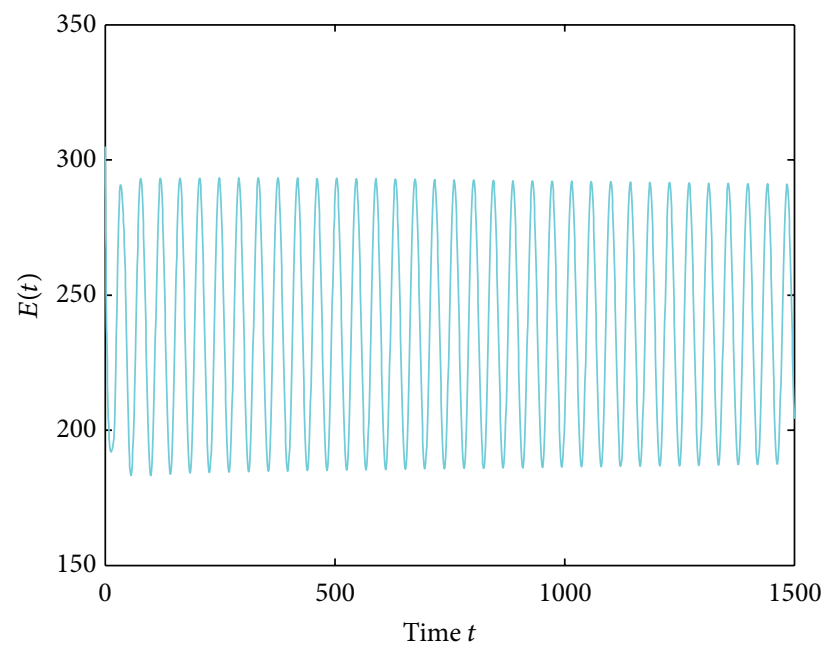

(b)

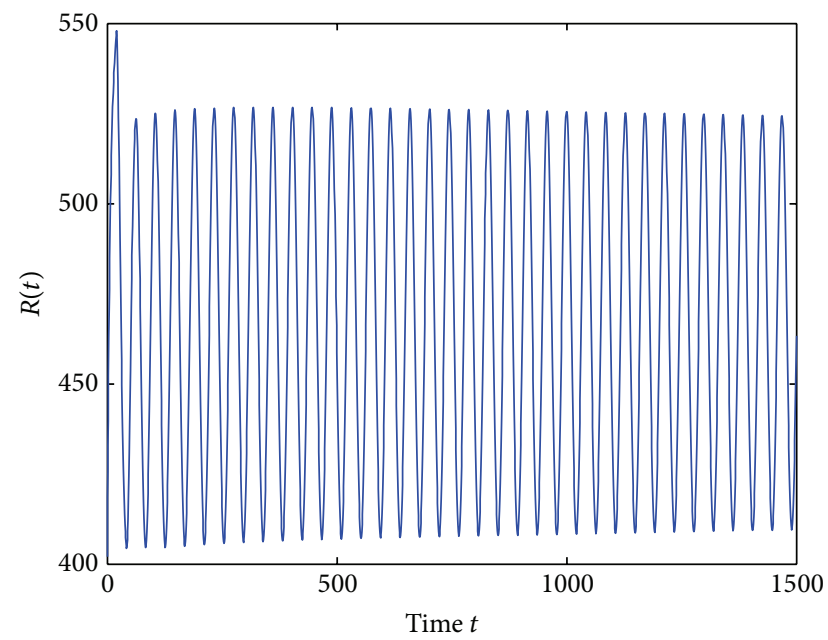

(d)

FIgURE 3: The track of the states $S, E, I$, and $R$ for $\tau_{1}=20.0500>17.2530=\tau_{10}$. 


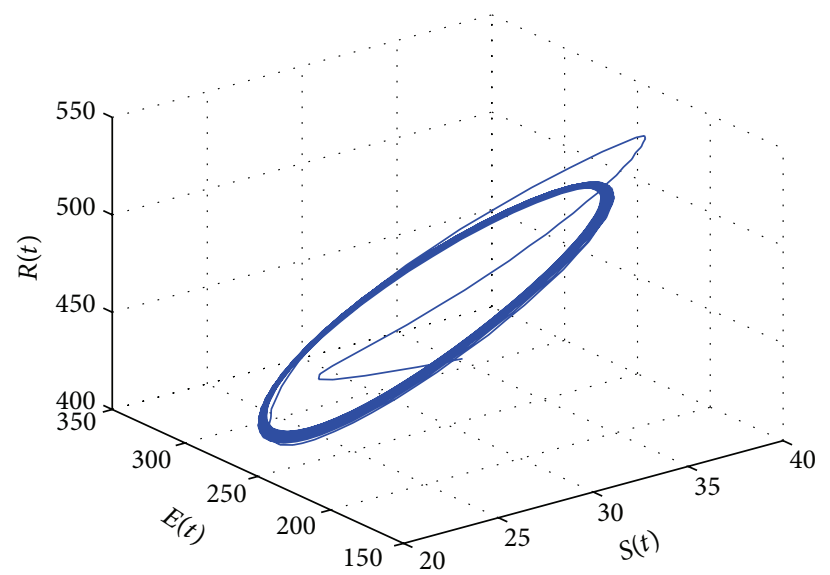

Figure 4: The phase plot of the states $S, E$, and $R$ for $\tau_{1}=20.0500>17.2530=\tau_{10}$.

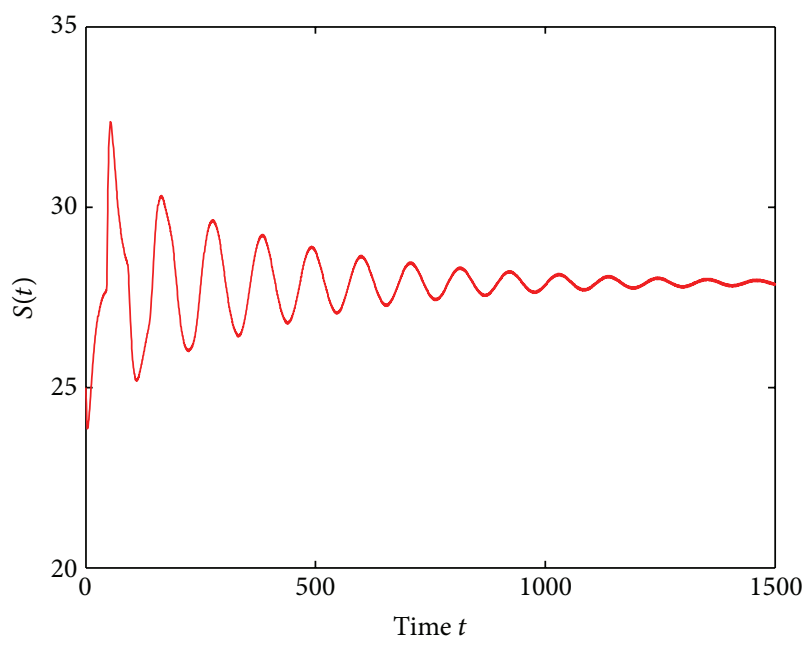

(a)

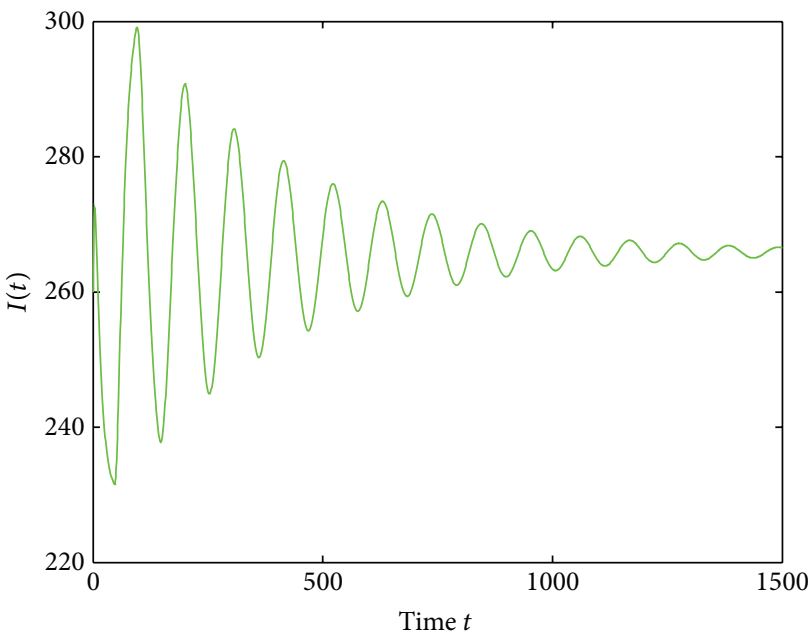

(c)

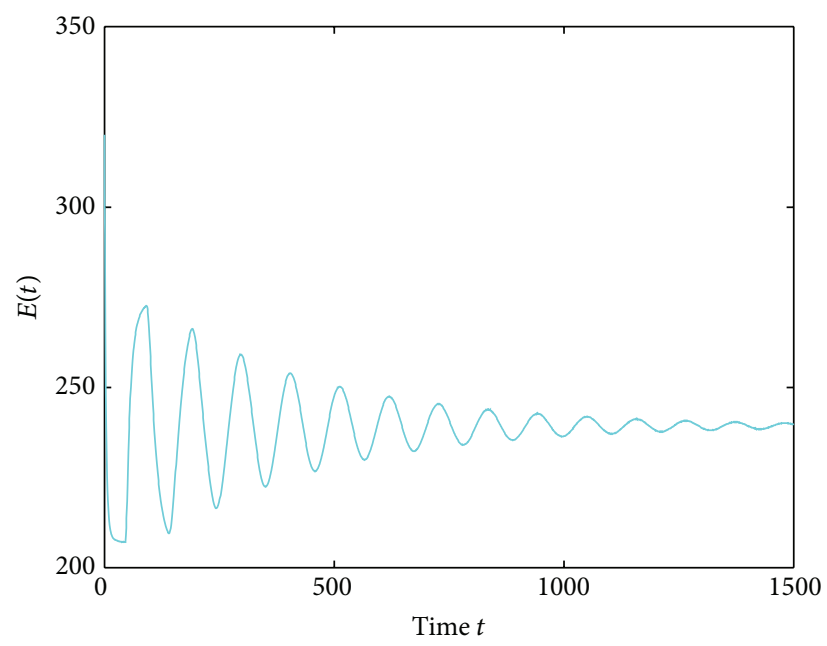

(b)

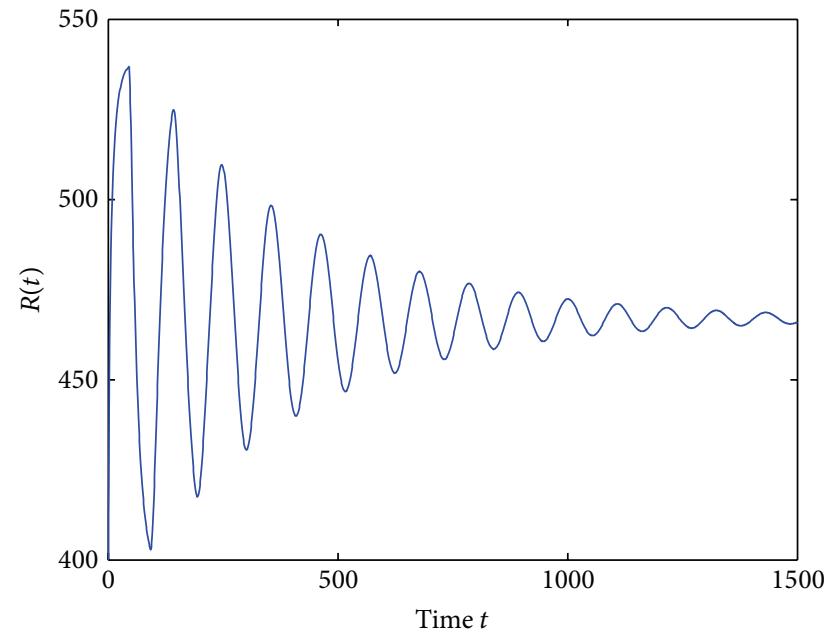

(d)

Figure 5: The track of the states $S, E, I$, and $R$ for $\tau_{2}=45.5000<52.4927=\tau_{20}$. 


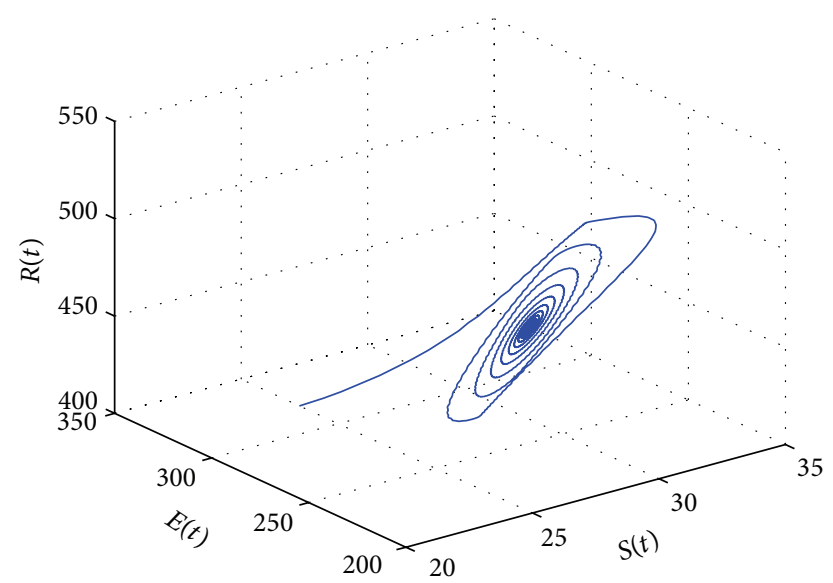

FIGURE 6: The phase plot of the states $S, E$, and $R$ for $\tau_{2}=45.5000<52.4927=\tau_{20}$.

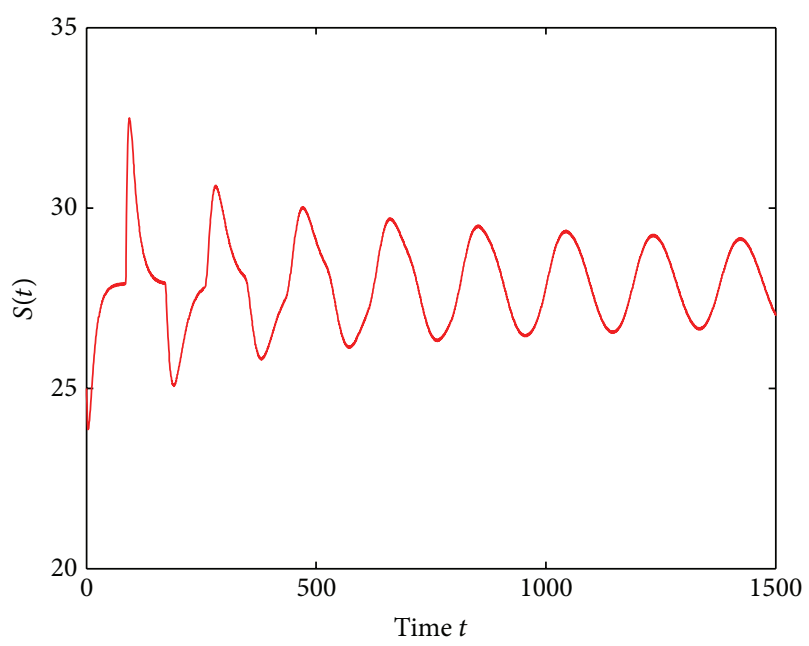

(a)

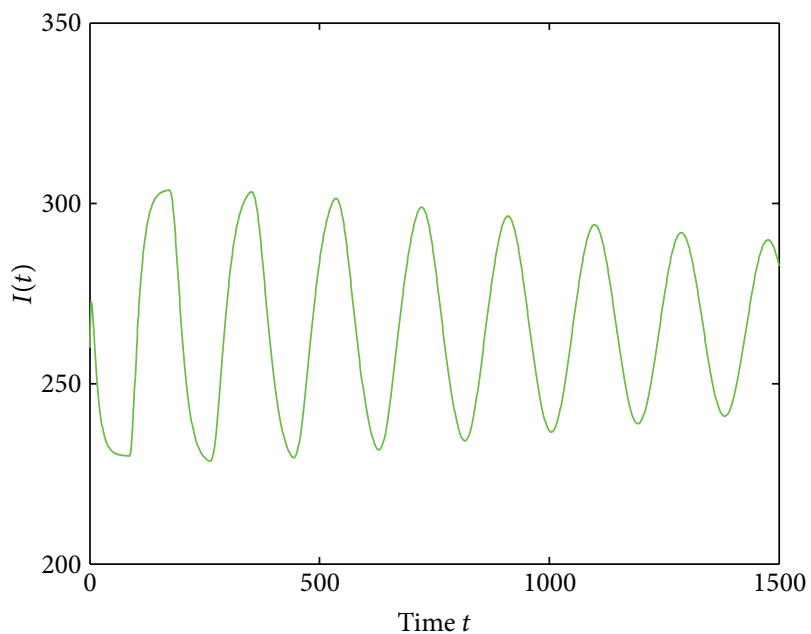

(c)

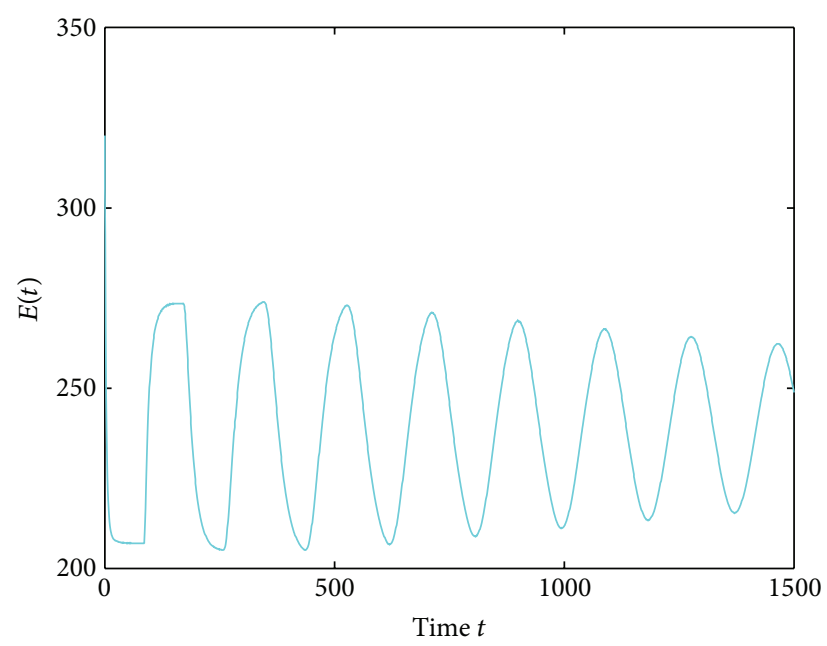

(b)

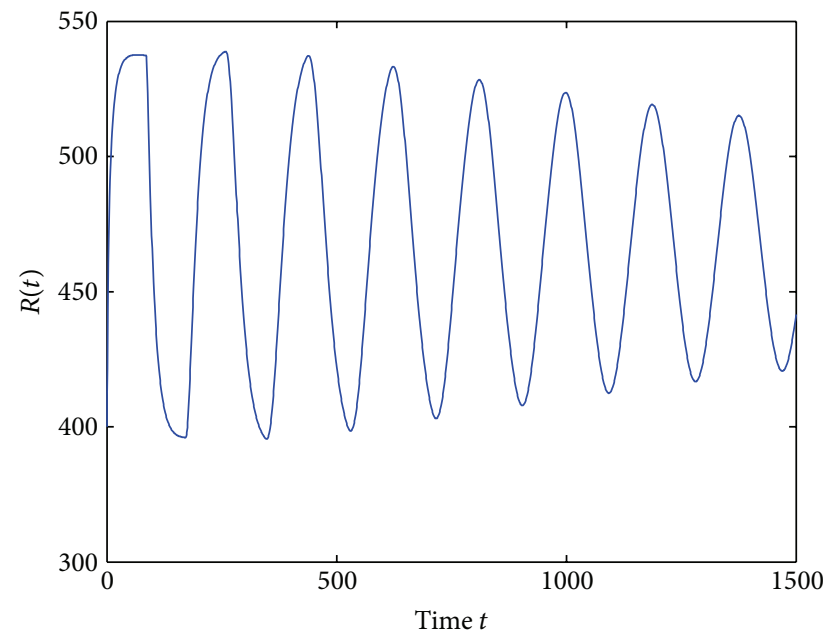

(d)

FigURE 7: The track of the states $S, E, I$, and $R$ for $\tau_{2}=67.5000>52.4927=\tau_{20}$. 


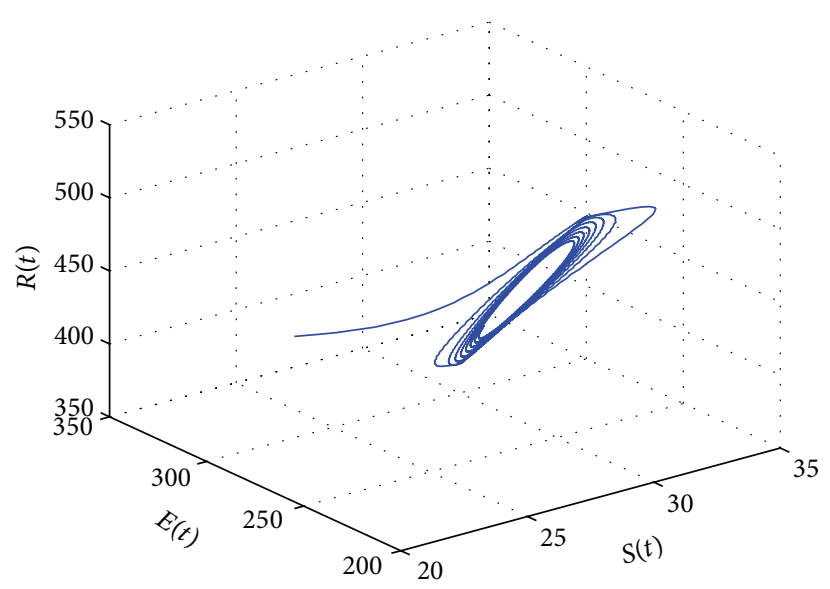

Figure 8: The phase plot of the states $S, E$, and $R$ for $\tau_{2}=67.5000>52.4927=\tau_{20}$.

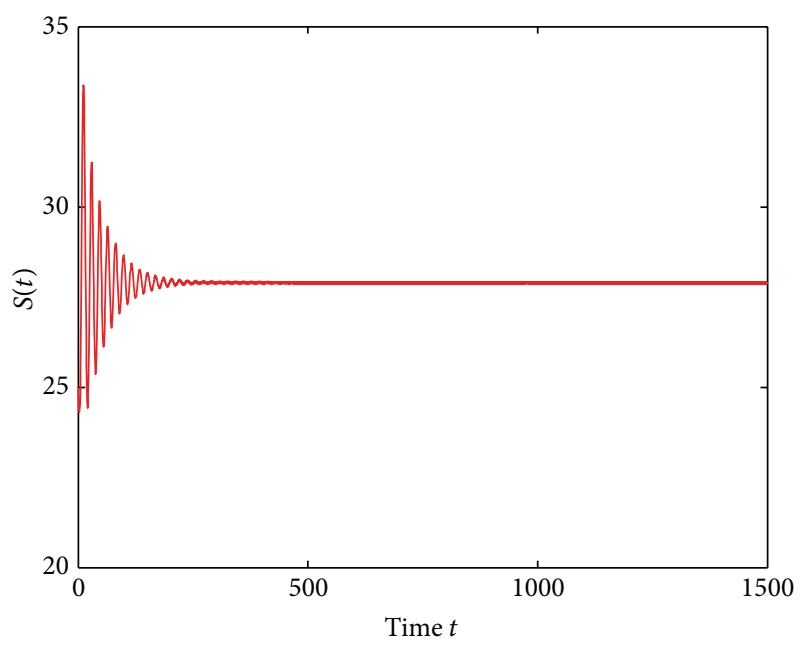

(a)

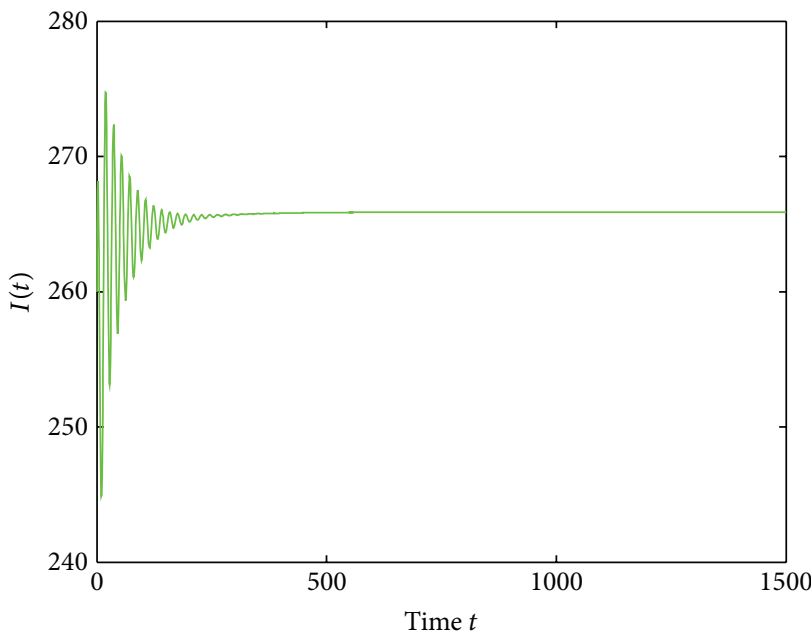

(c)

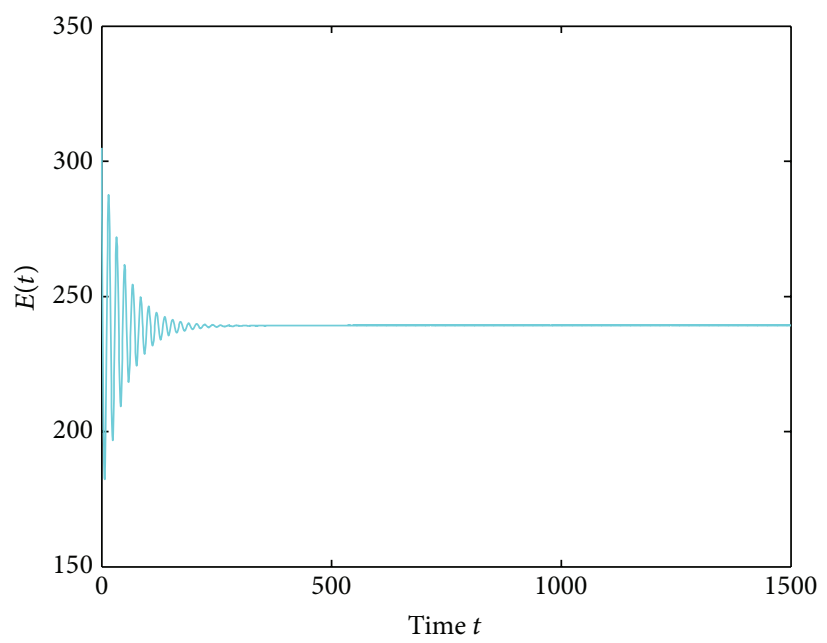

(b)

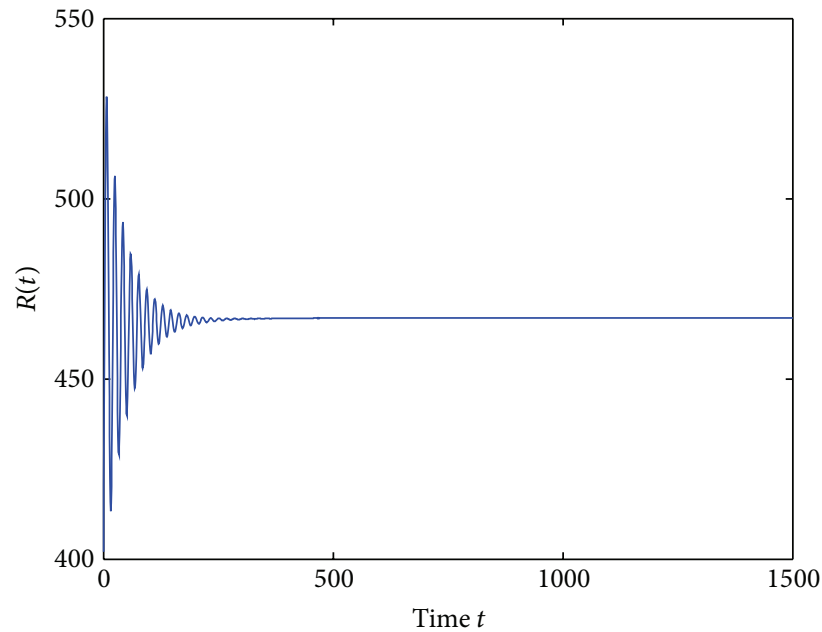

(d)

FIgURE 9: The track of the states $S, E, I$, and $R$ for $\tau=4.7500<5.1094=\tau_{0}$. 


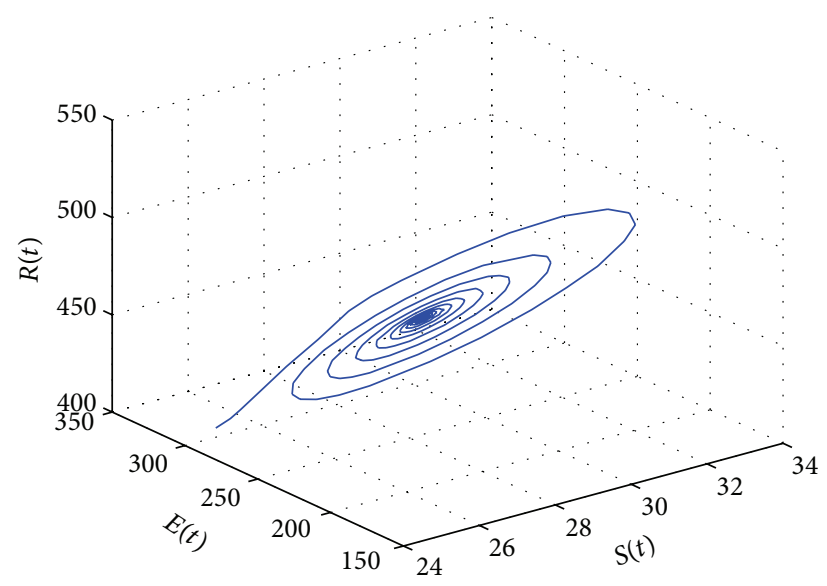

Figure 10: The phase plot of the states $S, E$, and $R$ for $\tau=4.7500<5.1094=\tau_{0}$.

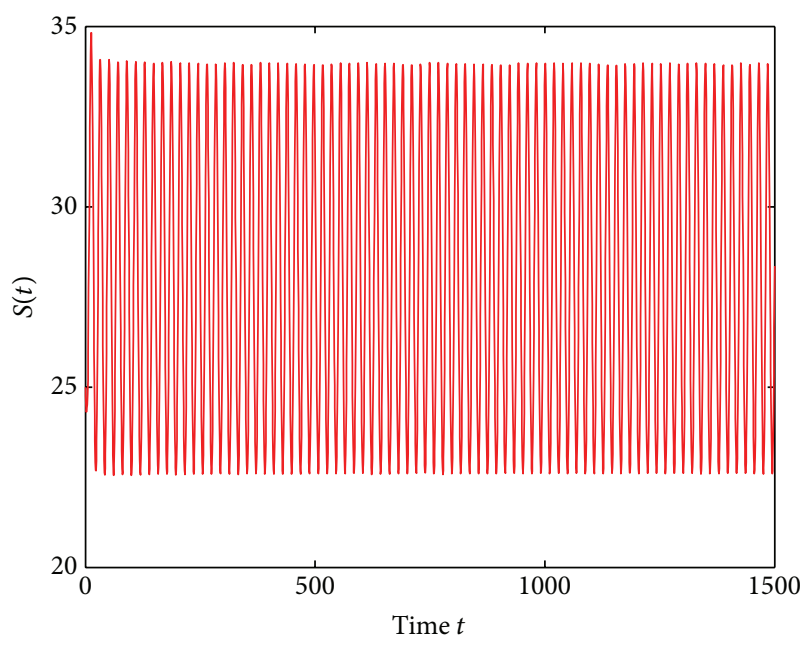

(a)

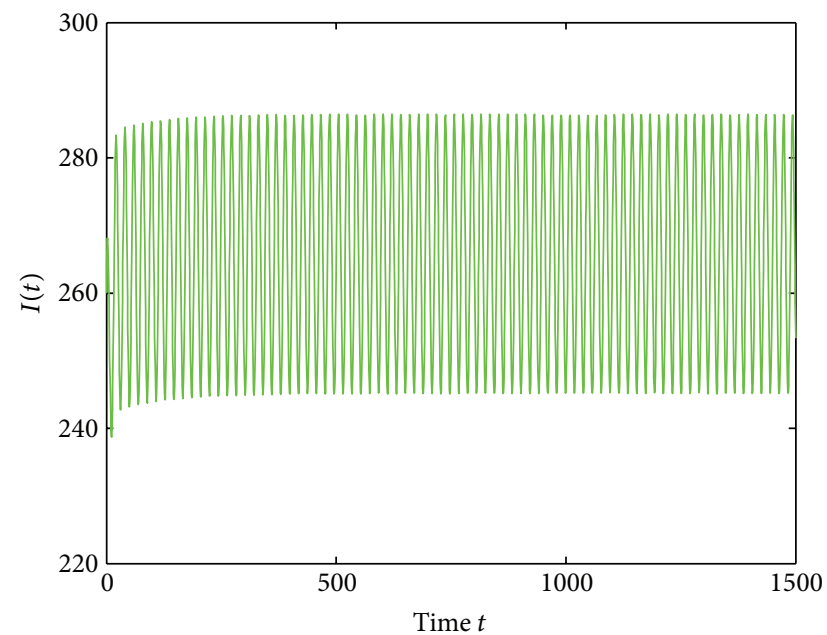

(c)

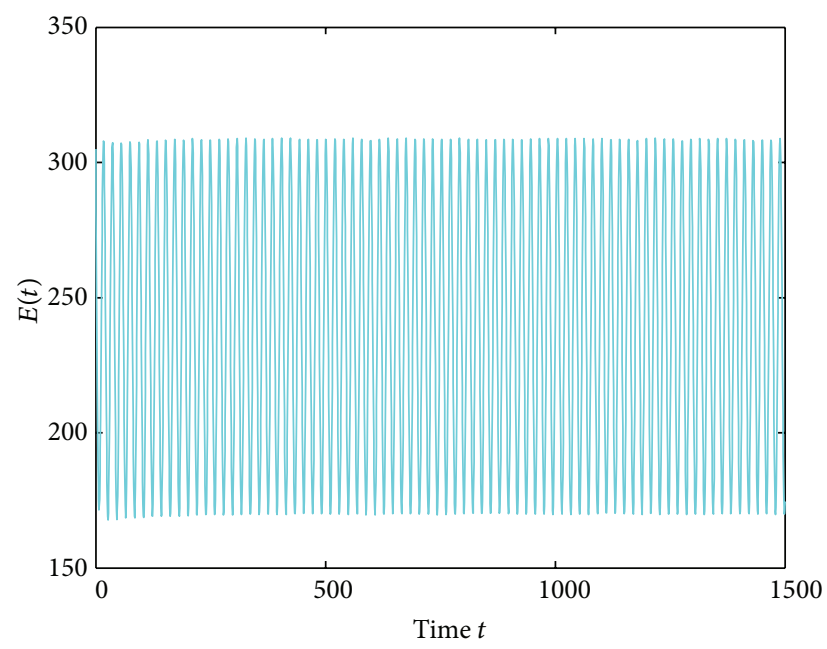

(b)

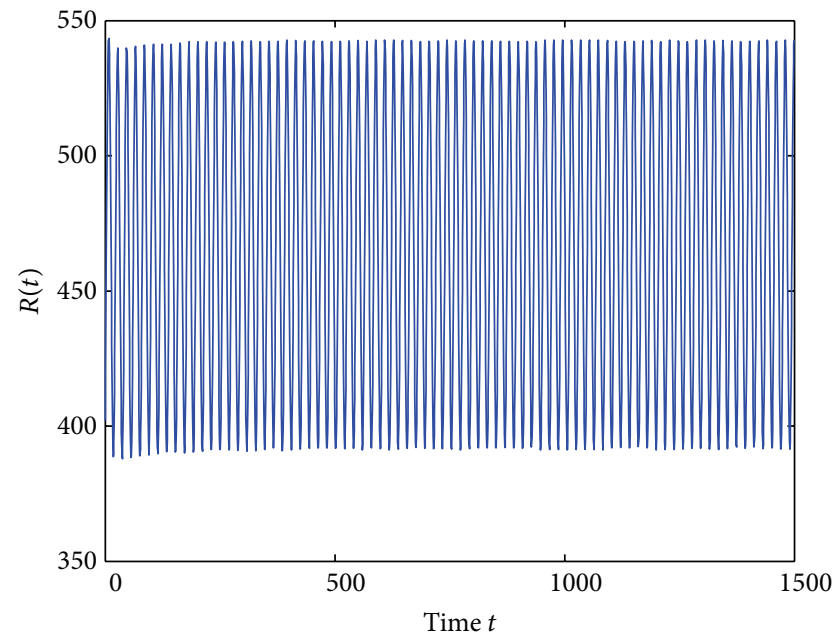

(d)

FIGURE 11: The track of the states $S, E, I$, and $R$ for $\tau=5.2300>5.1094=\tau_{0}$. 


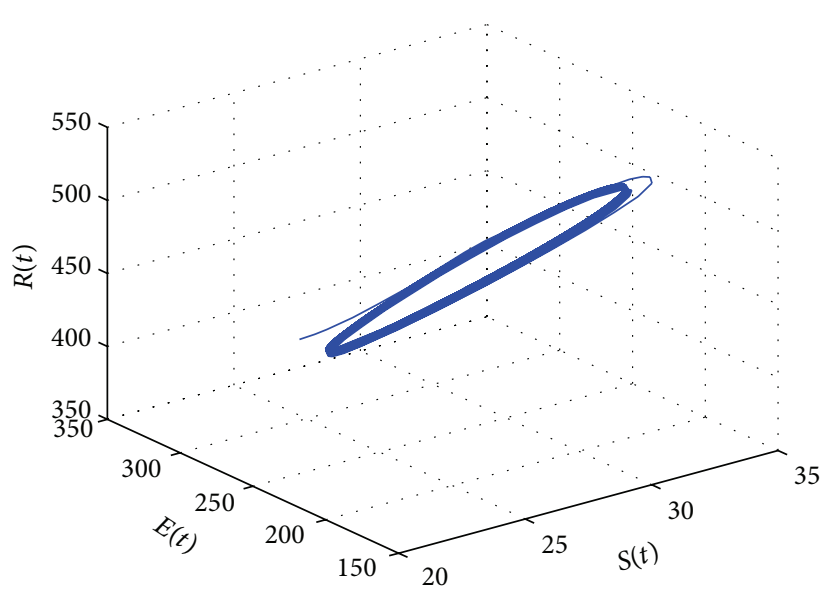

Figure 12: The phase plot of the states $S, E$, and $R$ for $\tau=5.2300>5.1094=\tau_{0}$.

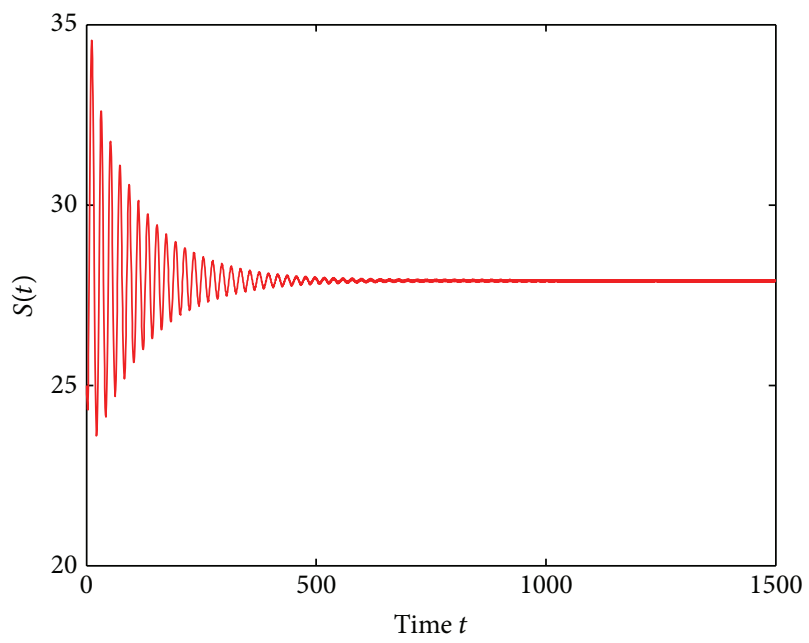

(a)

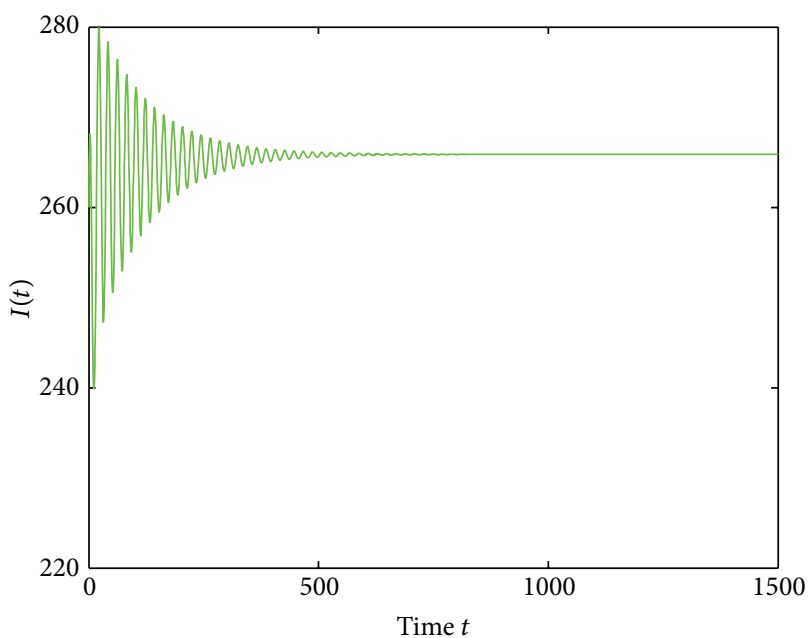

(c)

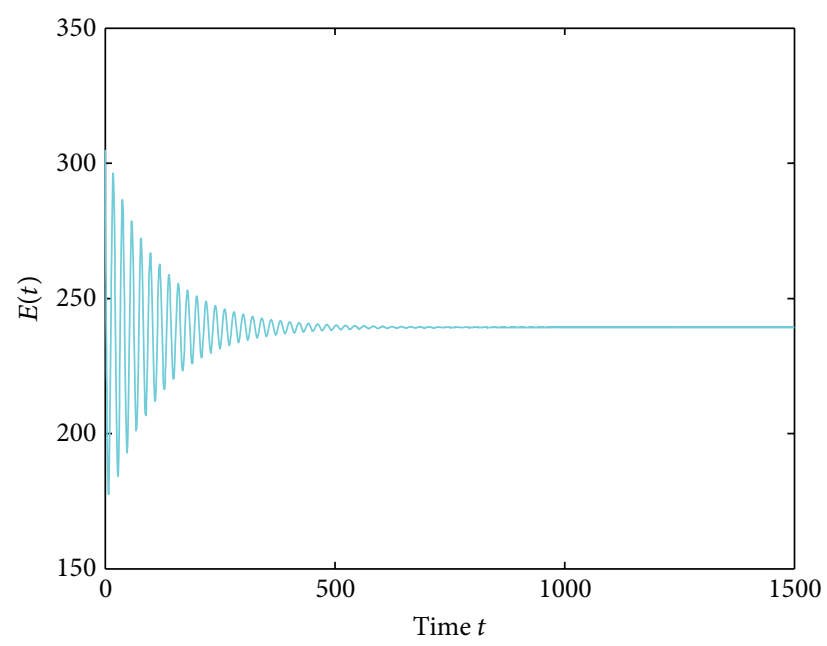

(b)

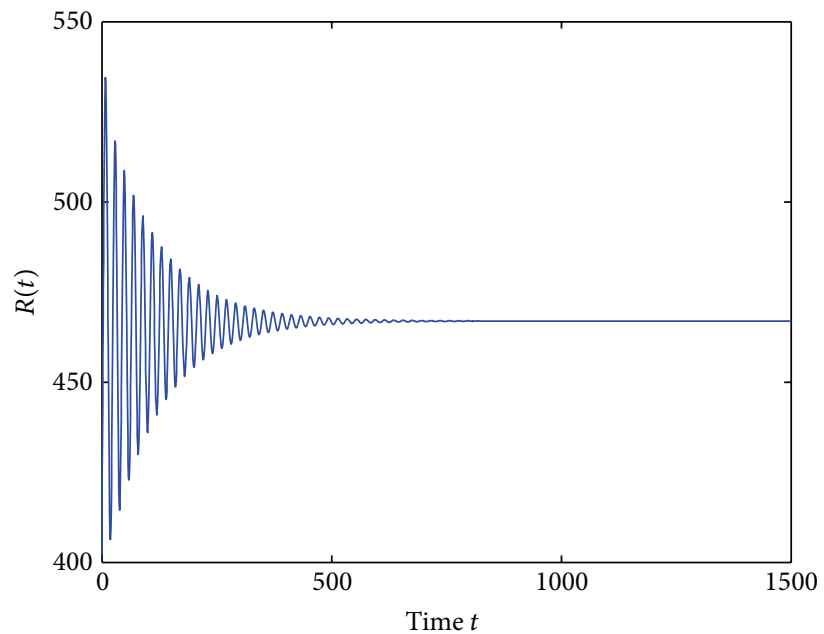

(d)

FIgURE 13: The track of the states $S, E, I$, and $R$ for $\tau_{1}=7.2000<7.8533=\tau_{1}^{*}$ and $\tau_{2}^{*}=3$. 


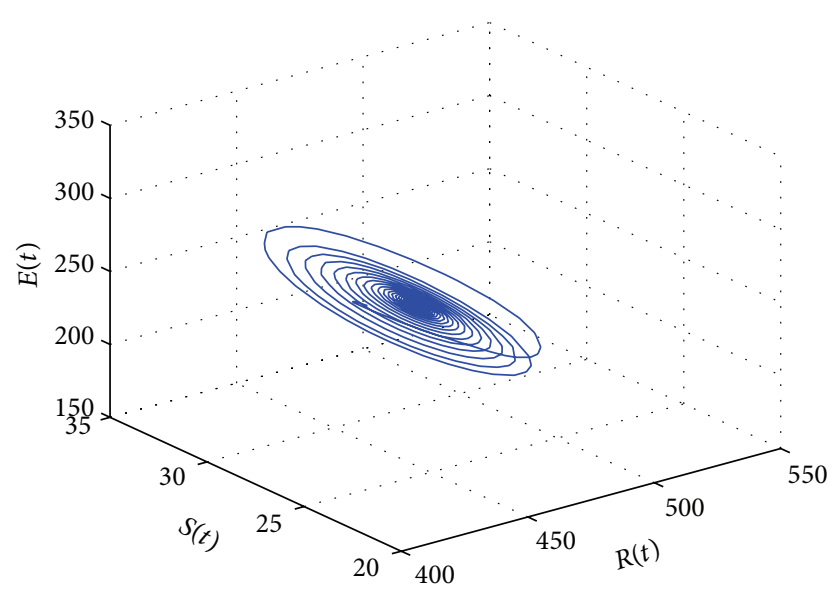

Figure 14: The phase plot of the states $R, S$, and $E$ for $\tau_{1}=7.2000<7.8533=\tau_{1}^{*}$ and $\tau_{2}^{*}=3$.

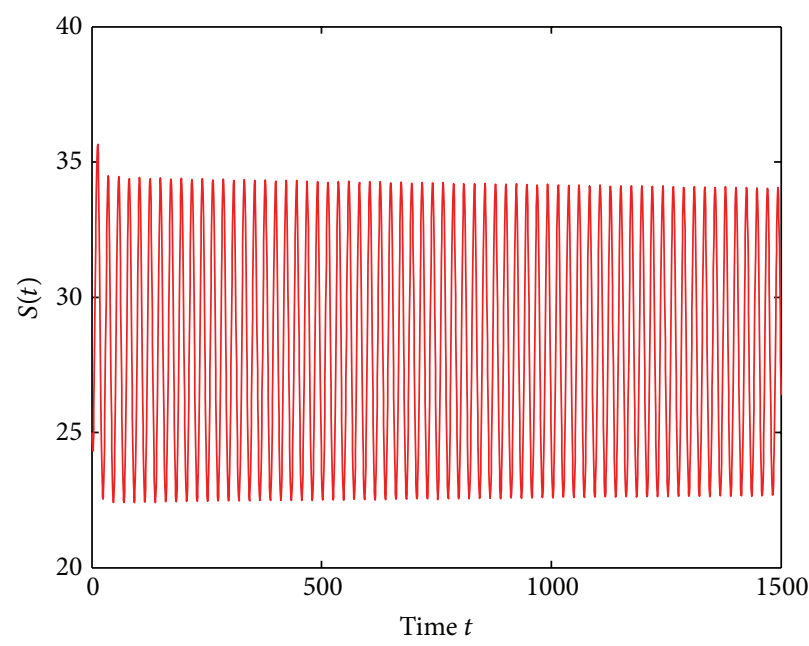

(a)

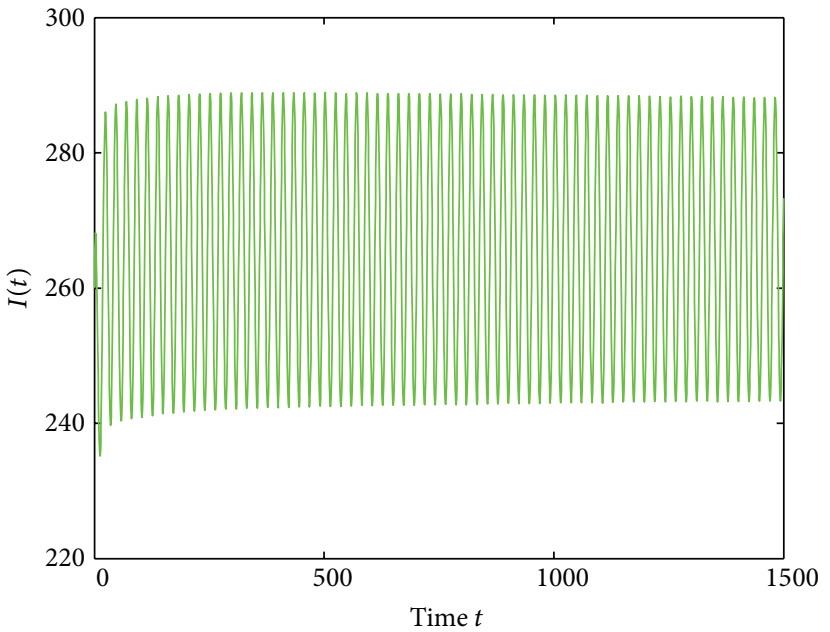

(c)

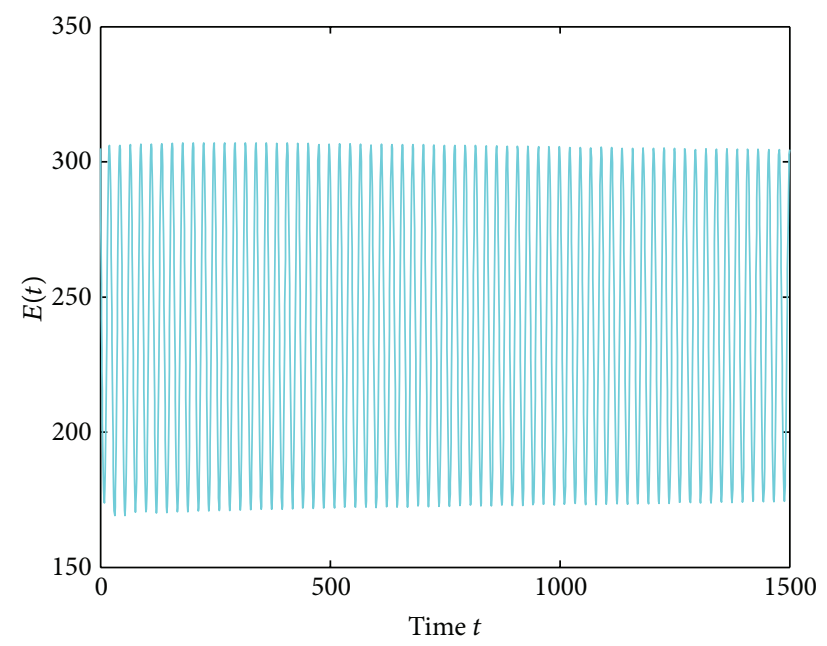

(b)

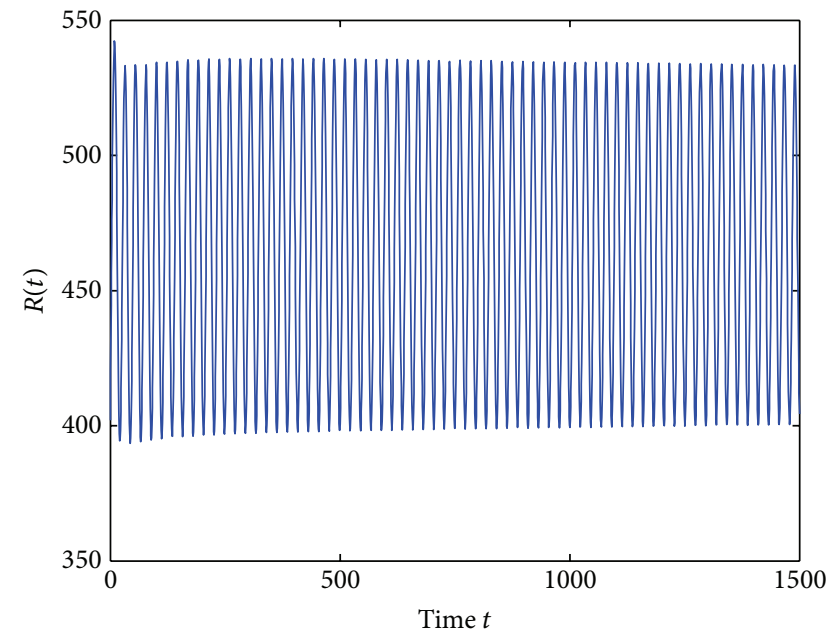

(d)

FIGURE 15: The track of the states $S, E, I$, and $R$ for $\tau_{1}=8.4500>7.8533=\tau_{1}^{*}$ and $\tau_{2}^{*}=3$. 


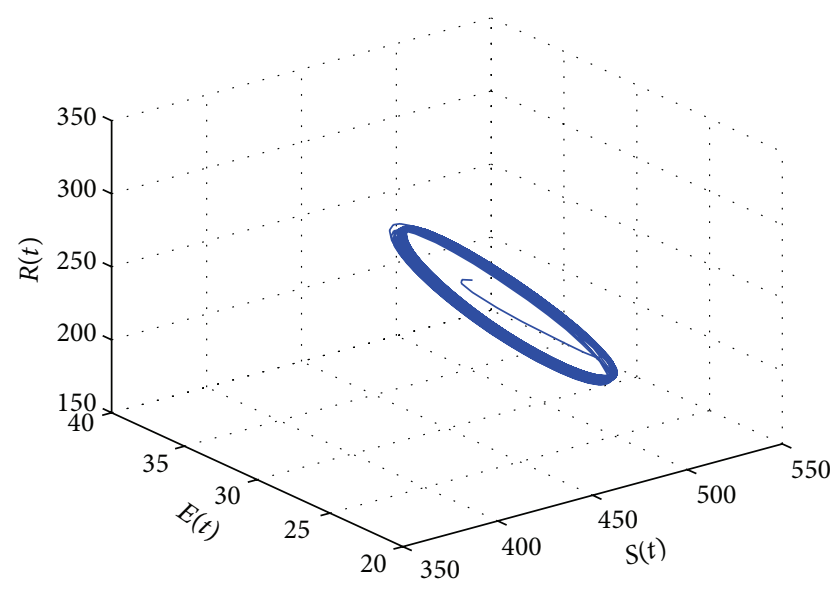

Figure 16: The phase plot of the states $R, S$, and $E$ for $\tau_{1}=8.4500>$ $7.8533=\tau_{1}^{*}$ and $\tau_{2}^{*}=3$.

\section{Conflict of Interests}

The authors declare that there is no conflict of interests regarding the publication of this article.

\section{Acknowledgment}

This work was supported by the National Natural Science Foundation of China (61273070), a project funded by the Priority Academic Program Development of Jiangsu Higher Education Institutions.

\section{References}

[1] J. O. Kephart and S. R. White, "Directed-graph epidemiological models of computer viruses," in Proceedings of the IEEE Computer Society Symposium on Research in Security and Privacy, pp. 343-359, May 1991.

[2] J. O. Kephart and S. R. White, "Measuring and modeling computer virus prevalence," in Proceedings of the IEEE Computer Society Symposium on Research in Security and Privacy, pp. 2-15, May 1993.

[3] J. C. Wierman and D. J. Marchette, "Modeling computer virus prevalence with a susceptible-infected-susceptible model with reintroduction," Computational Statistics \& Data Analysis, vol. 45, no. 1, pp. 3-23, 2004.

[4] B. K. Mishra and N. Jha, "Fixed period of temporary immunity after run of anti-malicious software on computer nodes," Applied Mathematics and Computation, vol. 190, no. 2, pp. 12071212, 2007.

[5] J. R. C. Piqueira and V. O. Araujo, "A modified epidemiological model for computer viruses," Applied Mathematics and Computation, vol. 213, no. 2, pp. 355-360, 2009.

[6] X. Han and Q. L. Tan, "Dynamical behavior of computer virus on internet," Applied Mathematics and Computation, vol. 217, no. 6, pp. 2520-2526, 2010.

[7] J. G. Ren, X. F. Yang, L.-X. Yang, Y. Xu, and F. Yang, "A delayed computer virus propagation model and its dynamics," Chaos, Solitons \& Fractals, vol. 45, no. 1, pp. 74-79, 2012.
[8] J. G. Ren, X. F. Yang, Q. Zhu, L.-X. Yang, and C. Zhang, "A novel computer virus model and its dynamics," Nonlinear Analysis. Real World Applications, vol. 13, no. 1, pp. 376-384, 2012.

[9] H. Yuan and G. Chen, "Network virus-epidemic model with the point-to-group information propagation," Applied Mathematics and Computation, vol. 206, no. 1, pp. 357-367, 2008.

[10] T. Dong, X. F. Liao, and H. Q. Li, "Stability and Hopf bifurcation in a computer virus model with multistate antivirus," Abstract and Applied Analysis, vol. 2012, Article ID 841987, 16 pages, 2012.

[11] B. K. Mishra and D. K. Saini, "SEIRS epidemic model with delay for transmission of malicious objects in computer network," Applied Mathematics and Computation, vol. 188, no. 2, pp. 14761482, 2007.

[12] B. K. Mishra and S. K. Pandey, "Dynamic model of worms with vertical transmission in computer network," Applied Mathematics and Computation, vol. 217, no. 21, pp. 8438-8446, 2011.

[13] F. W. Wang, Y. K. Zhang, C. G. Wang, J. Ma, and S. Moon, "Stability analysis of a SEIQV epidemic model for rapid spreading worms," Computers and Security, vol. 29, no. 4, pp. 410-418, 2010.

[14] Y. Yao, X.-W. Xie, H. Guo, G. Yu, F.-X. Gao, and X.-J. Tong, "Hopf bifurcation in an Internet worm propagation model with time delay in quarantine," Mathematical and Computer Modelling, vol. 57, pp. 2635-2646, 2013.

[15] T. L. Zhang, J. L. Liu, and Z. D. Teng, "Stability of Hopf bifurcation of a delayed SIRS epidemic model with stage structure," Nonlinear Analysis. Real World Applications, vol. 11, no. 1, pp. 293-306, 2010.

[16] S. Gakkhar and A. Singh, "Complex dynamics in a prey predator system with multiple delays," Communications in Nonlinear Science and Numerical Simulation, vol. 17, no. 2, pp. 914-929, 2012.

[17] X.-Y. Meng, H.-F. Huo, and H. Xiang, "Hopf bifurcation in a three-species system with delays," Journal of Applied Mathematics and Computing, vol. 35, no. 1-2, pp. 635-661, 2011.

[18] T. K. Kar and A. Ghorai, "Dynamic behaviour of a delayed predator-prey model with harvesting," Applied Mathematics and Computation, vol. 217, no. 22, pp. 9085-9104, 2011.

[19] J.-F. Zhang, "Bifurcation analysis of a modified Holling-Tanner predator-prey model with time delay," Applied Mathematical Modelling, vol. 36, no. 3, pp. 1219-1231, 2012.

[20] S. Guo and W. Jiang, "Global stability and Hopf bifurcation for Gause-type predator-prey system," Journal of Applied Mathematics, vol. 2012, Article ID 260798, 17 pages, 2012.

[21] S. Jana and T. K. Kar, "Modeling and analysis of a prey-predator system with disease in the prey," Chaos, Solitons \& Fractals, vol. 47, pp. 42-53, 2013.

[22] C. J. Xu and X. F. He, "Stability and bifurcation analysis in a class of two-neuron networks with resonant bilinear terms," Abstract and Applied Analysis, vol. 2011, Article ID 697630, 21 pages, 2011.

[23] Y. K. Li and C. Z. Li, "Stability and Hopf bifurcation analysis on a delayed Leslie-Gower predator-prey system incorporating a prey refuge," Applied Mathematics and Computation, vol. 219, no. 9, pp. 4576-4589, 2013.

[24] B. D. Hassard, N. D. Kazarinoff, and Y. H. Wan, Theory and Applications of Hopf Bifurcation, Cambridge University Press, Cambridge, UK, 1981. 


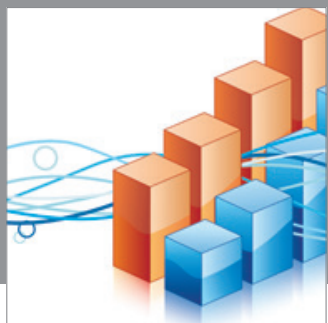

Advances in

Operations Research

mansans

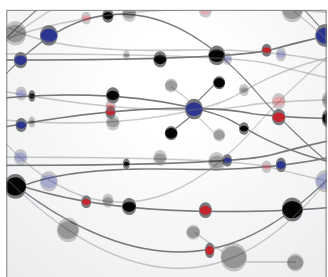

The Scientific World Journal
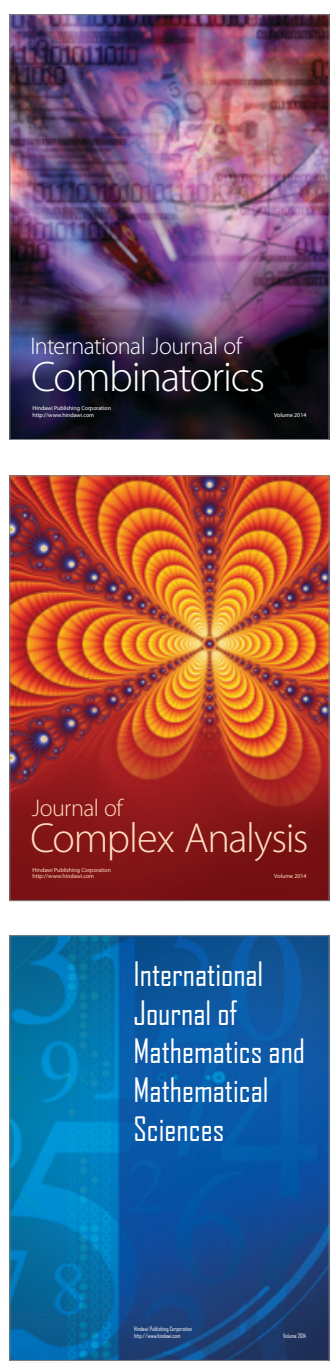
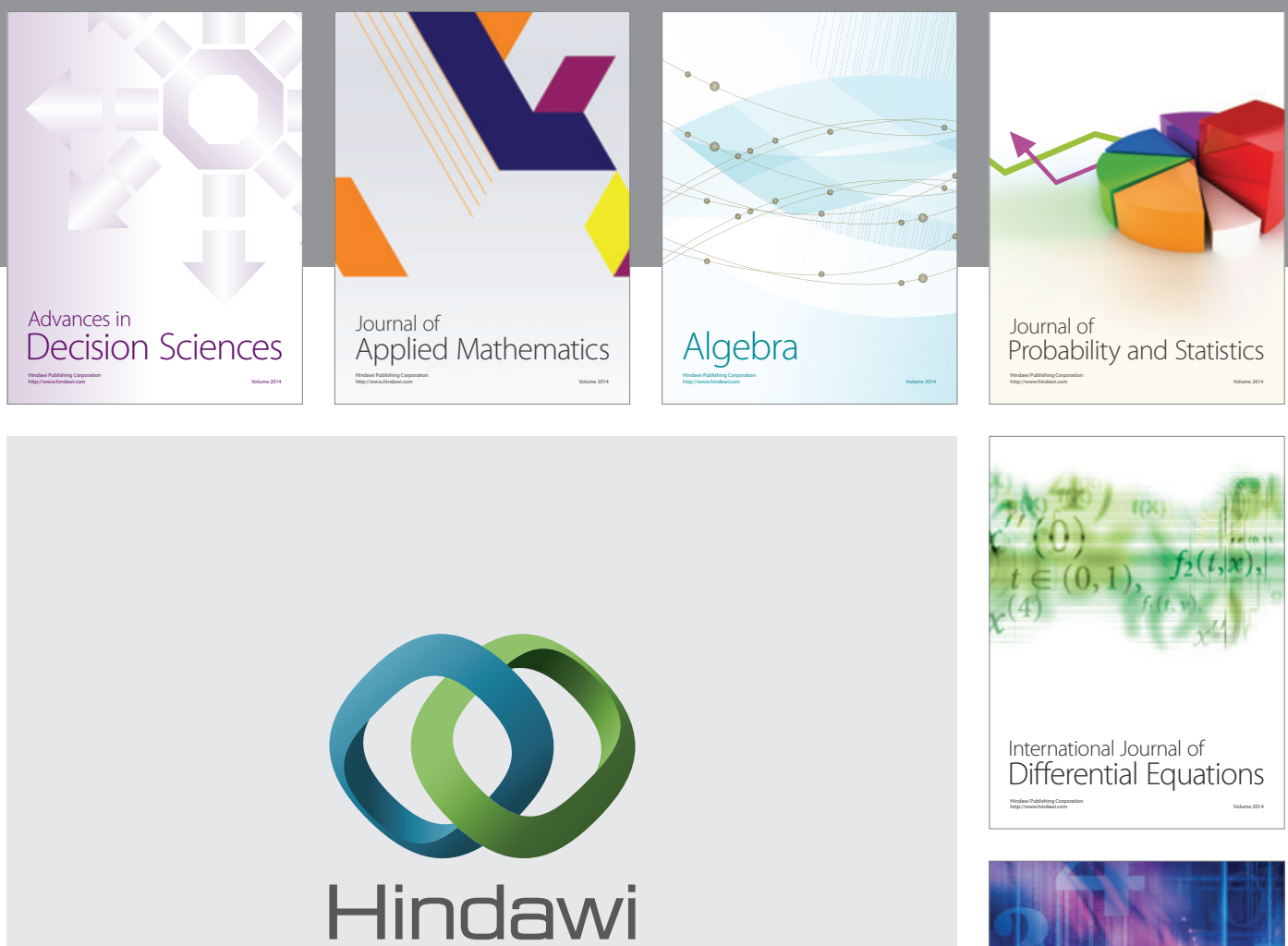

Submit your manuscripts at http://www.hindawi.com
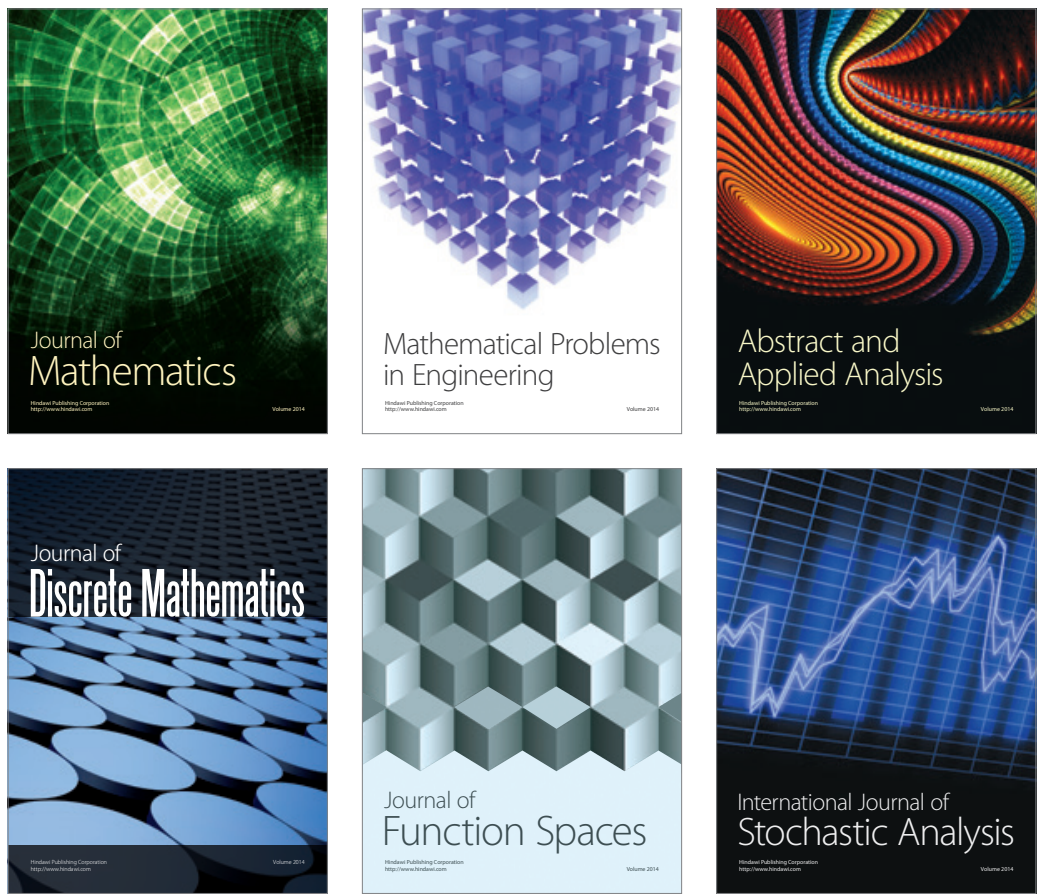

Journal of

Function Spaces

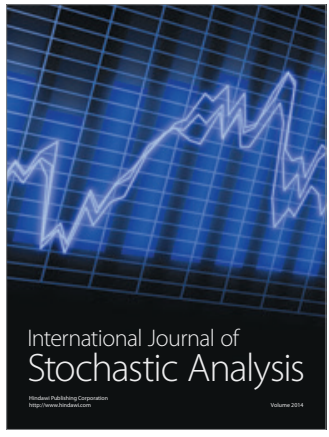

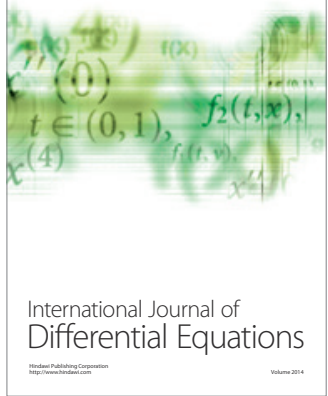
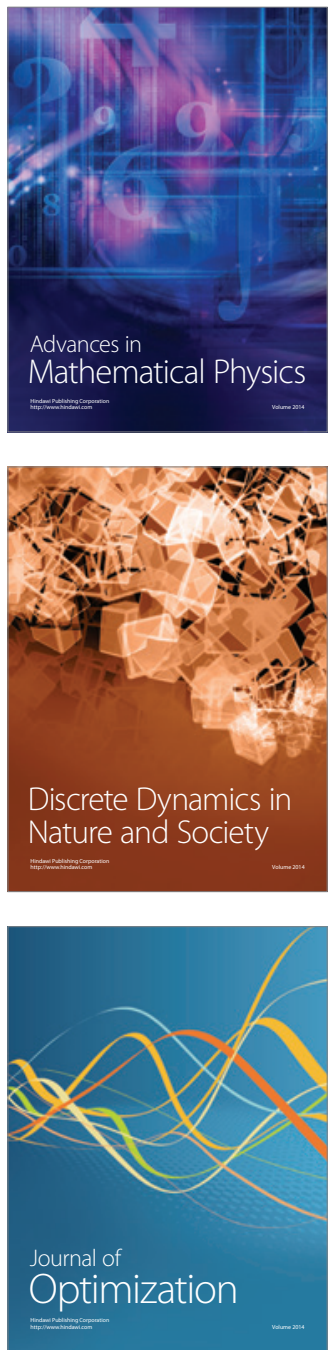